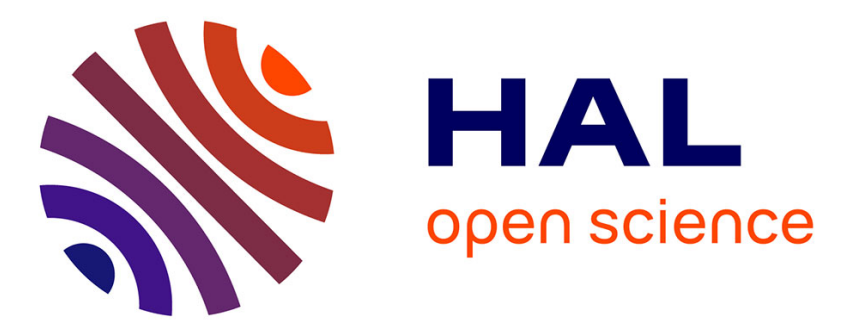

\title{
Adaptive fuzzy controller for multivariable nonlinear state time-varying delay systems subject to input nonlinearities
}

\author{
Abdesselem Boulkroune, Mohammed M’Saad, Mondher Farza
}

\section{To cite this version:}

Abdesselem Boulkroune, Mohammed M'Saad, Mondher Farza. Adaptive fuzzy controller for multivariable nonlinear state time-varying delay systems subject to input nonlinearities. Fuzzy Sets and Systems, 2011, 164 (1), pp.Pages 45-65. 10.1016/j.fss.2010.09.001 . hal-01060374

\section{HAL Id: hal-01060374 https://hal.science/hal-01060374}

Submitted on 10 Sep 2014

HAL is a multi-disciplinary open access archive for the deposit and dissemination of scientific research documents, whether they are published or not. The documents may come from teaching and research institutions in France or abroad, or from public or private research centers.
L'archive ouverte pluridisciplinaire HAL, est destinée au dépôt et à la diffusion de documents scientifiques de niveau recherche, publiés ou non, émanant des établissements d'enseignement et de recherche français ou étrangers, des laboratoires publics ou privés. 


\title{
Adaptive fuzzy controller for multivariable nonlinear state time-varying delay systems subject to input nonlinearities
}

\author{
A. Boulkroune ${ }^{\mathrm{a}, *}$, M. M'Saad ${ }^{\mathrm{b}}$, M. Farza ${ }^{\mathrm{b}}$ \\ a Department of Automatic, Faculty of Engineering Sciences, University of Jijel, BP. 98, OuledAissa, 18000 Jijel, Algeria \\ b GREYC, UMR 6072 CNRS, Université de Caen, ENSICAEN, 6 Bd Maréchal Juin, 14050 Caen Cedex, France
}

\begin{abstract}
In this paper, an adaptive fuzzy variable-structure controller is investigated for a class of uncertain multi-input multi-output (MIMO) nonlinear time-delay systems with both sector nonlinearities and dead-zones. A decomposition property of the control-gain matrix is fully exploited in the controller design and the stability analysis. The unknown time-varying delay uncertainties are compensated for using an appropriate Lyapunov-Krasovskii functional. The boundedness of all signals of the closed-loop system as well as the exponential convergence of the underlying tracking errors to an adjustable region are established. The effectiveness of the proposed fuzzy adaptive controller is illustrated throughout simulation results.
\end{abstract}

(C) 2010 Elsevier B.V. All rights reserved.

Keywords: Adaptive control; Variable-structure control; Fuzzy system; MIMO nonlinear systems; Time-delay; Sector nonlinearity; Dead-zone

\section{Introduction}

Fuzzy systems (FSs) have been successfully applied to many control problems because they do not need an accurate mathematical model of the system under control and they can cooperate with human expert knowledge. It is also known that FSs as well as neural networks (NNs) can approximate uniformly any nonlinear continuous function over a compact set [33]. Thanks to the universal approximation theorem [33], adaptive fuzzy control schemes $[3,5,6,9,13,21,22,24,29-31]$ have been developed for a class of MIMO nonlinear uncertain systems. The stability of the underlying closed-loop system has been analyzed using a Lyapunov approach. To cope with the ubiquitous fuzzy approximation error and external disturbances, these adaptive fuzzy controllers are augmented by a robust compensator, namely a sliding mode control $[3,6,13,21,24,30,31]$ or an $H^{\infty}$ control $[5,9,22,29]$. A key assumption in all previous fuzzy adaptive control schemes [3,5,6,9,13,21,22,24,29-31] is that the considered MIMO systems are characterised by linear inputs, i.e. the actuator dynamics may be reasonably approximated by a linear model which is more an exception than a rule in the engineering practice.

The control problem of uncertain multivariable systems with nonlinear input channels has received a remarkable attention because of those ubiquitous actuator nonlinearities, namely saturation, quantization, backlash, dead-zones and

\footnotetext{
* Corresponding author.

E-mail addresses: boulkroune2002@yahoo.fr (A. Boulkroune), msaad@greyc.ensicaen.fr (M. M’Saad), mfarza@greyc.ensicaen.fr (M. Farza).
} 
so on $[15,17]$. It is well known that the existence of input nonlinearities may lead to notable performance degradations or even instability of the control system. It is thereby more advisable to take into account the actuator nonlinearities in the control design as well as the stability analysis. Decentralized variable-structure controllers have been proposed in $[16,25,26]$ for a class of systems with input sector nonlinearities and/or dead-zones. In $[17,23]$, the authors designed sliding mode control systems for nonlinear multivariable systems subject to both sector nonlinearities and dead-zones. The underlying results suffer from two fundamental limitations. Firstly, the considered class of the systems is relatively reduced. Secondly, the gain reduction tolerances of the nonlinear dead-zones and upper bounds of the uncertain nonlinear functions are required to be known.

More recently, adaptive neural and fuzzy control systems have been, respectively, proposed for a particular class of multivariable nonlinear systems with unknown dead-zones and gain signs in $[34,35]$. These contributions suffer from two restrictive modelling assumptions motivated by technical purposes regarding the stability analysis and control design. The first one consists in assuming a lower triangular control structure for the system under control while the second one concerns the boundedness of the high-frequency control gains. Moreover, an adaptive fuzzy control for a class of MIMO nonlinear systems with unknown dead-zones has been designed in [1]. Note that, in [1,34,35], simple dead-zones having linear and nonlinear functions outside the dead-band have been considered.

Time-delay is frequently encountered in various engineering systems, such as chemical processes, electrical networks, nuclear reactor, manual control and long transmission lines in pneumatic, hydraulic systems and rolling mill systems, etc. The existence of the time-delays may be the source of instability and may degrade the performance of the closedloop system. A remarkable research activity has been devoted to neural and fuzzy adaptive control for time-delay nonlinear systems. The corresponding contributions heavily borrow from the robust stability analysis approaches that have been developed for time-delay linear systems over the last years $[14,19,20,28]$. The seminal contributions have been devoted to the adaptive neural control for single-input single-output nonlinear time-delay systems in strict-feedback form $[10,11]$. Though the underlying control systems are intrinsically robust with respect to the uncertainties resulting from unknown time-delays, they suffer from the applicability point of view. Indeed, some restrictions on the functions of delayed states have been introduced for technical considerations imposed by the adopted Lyapunov control design approach. More important contributions have made for multivariable nonlinear time-delay systems in block-triangular form using the adaptive neural control [12,34]. In [12], the neural networks are essentially used to handle the unknown functions in systems dynamics as well as the unknown bounds of the functions of delayed states. Unlike in $[10,11]$, the restrictive assumption on the function of delayed states has been removed thanks to a useful separation technique. The contribution [34] consists in designing an adaptive neural controller that has been proposed for nonlinear state time-varying delay systems in triangular control structure with unknown nonlinear dead-zone and gain sign. The control design is particularly inspired from the fundamental developments in $[10,11]$ and share henceforth the same limitations. A fuzzy adaptive controller, inspired from [10,11], has been recently proposed in [32] for nonlinear time-delay systems with unknown virtual control coefficients. Although an upper bound of time-varying delay functions is assumed to be known, only one parameter is tuned by the involved learning law.

Motivated by the fundamental results in [1,17,23,34,35], one aims at designing a fuzzy adaptive controller for a class of MIMO uncertain nonlinear time-varying delays systems containing both sector nonlinearities and dead-zones in input.

Compared with the available results [17,23], the main contributions of our work include:

- The considered class of the MIMO systems is relatively large.

- The knowledge of gain reduction tolerances of the nonlinear dead-zones and upper bounds of the uncertain nonlinear functions is no longer required in the control design. Indeed, the upper bounds of the underlying functions are estimated using the fuzzy adaptive systems.

- The control-gain matrix is appropriately decomposed into a product of a symmetric definite-positive matrix, a diagonal matrix with +1 or -1 on the diagonal and a unity upper triangular matrix. It is worth noticing that the diagonal matrix elements are nothing than the ratios of the signs of the leading principal minors of the control-gain matrix. This matrix decomposition heavily borrows from $[1,3,7,8,18,36]$.

Compared also with [34,35], the main contributions of our work are:

- The modelling assumptions have been relaxed with respect to those in [34,35], namely lower triangular control structure with bounded high-frequency control gains. These modelling requirements were mainly motivated by stability analysis and control design purposes. 
- The considered model of the input nonlinearity includes sector nonlinearities and dead-zones and is hence relatively larger than the one considered in $[34,35]$.

- Unlike in [34], the proposed controllers have two important features that are worth to be pointed out. The first one is the singularity free control and the corresponding implementation feasibility. The second one is of practical interest as no prior knowledge on the time-varying delay is assumed. Notice that upper bounds on the time-delay and its derivative are commonly assumed to be known.

- The stability analysis is relatively simple and different from that pursued in [34]. Recall that the proof process of the stability in [34] is very complicated, as it requires the discussing of many cases.

\section{Notation, problem statement and preliminaries}

Consider the following class of uncertain nonlinear MIMO time-delay systems:

$$
\left\{\begin{array}{l}
y_{1}^{\left(r_{1}\right)}=f_{1}(x)+h_{\tau 1}\left(x_{\tau}\right)+\sum_{j=1}^{p} g_{1 j}(x) \Phi_{j}\left(u_{j}\right), \\
\vdots \\
y_{p}^{\left(r_{p}\right)}=f_{p}(x)+h_{\tau p}\left(x_{\tau}\right)+\sum_{j=1}^{p} g_{p j}(x) \Phi_{j}\left(u_{j}\right), \\
x_{i}(t)=\phi_{x_{i}}(t), t \in\left[-\tau_{\max }, 0\right], \quad \forall i=1, \ldots, p,
\end{array}\right.
$$

where $x=\left[x_{1}^{T}, \ldots, x_{p}^{T}\right]^{T} \in R^{r}$ is the overall state vector which is assumed available for measurement, with $x_{i}=$ $\left[y_{i}, \dot{y}_{i}, \ldots, y_{i}^{\left(r_{i}-1\right)}\right]^{T} \in R^{r_{i}}$ and $r=r_{1}+\cdots+r_{p}, x_{\tau}=\left[x_{1}^{T}\left(t-\tau_{1}(t)\right), \ldots, x_{p}^{T}\left(t-\tau_{p}(t)\right)\right]^{T} \in R^{r}$ is the delayed state vector, with $x_{\tau i}=x_{i}^{T}\left(t-\tau_{i}(t)\right)=\left[y_{i}\left(t-\tau_{i}(t)\right), \dot{y}_{i}\left(t-\tau_{i}(t)\right), \ldots, y_{i}^{\left(r_{i}-1\right)}\left(t-\tau_{i}(t)\right)\right]^{T} \in R^{r_{i}}, \forall i=1, \ldots, p$, $\tau_{1}(t), \ldots, \tau_{p}(t)$ are unknown time-varying delays, $\phi_{x_{1}}(t), \ldots, \phi_{x_{p}}(t)$ are known continuous initial state vector functions, $\tau_{\max }$ which will be defined later is an unknown positive constant. $u=\left[u_{1}, \ldots, u_{p}\right]^{T} \in R^{p}$ is the control input vector, $y=\left[y_{1}, \ldots, y_{p}\right]^{T} \in R^{p}$ is the output vector, and $f_{i}(x), h_{\tau i}\left(x_{\tau}\right), g_{i j}(x), i, j=1, \ldots, p$ are unknown continuous nonlinear functions, $\Phi(u)=\left[\Phi_{1}\left(u_{1}\right), \Phi_{2}\left(u_{2}\right), \ldots, \Phi_{p}\left(u_{p}\right)\right]^{T}$ is a nonlinear input function vector satisfying some properties which will be given later.

Let us denote

$$
\begin{aligned}
& y^{(r)}=\left[\begin{array}{lll}
y_{1}^{\left(r_{1}\right)} & \ldots & y_{p}^{\left(r_{p}\right)}
\end{array}\right]^{T}, \quad F(x)=\left[\begin{array}{lll}
f_{1}(x) & \ldots & f_{p}(x)
\end{array}\right]^{T}, \\
& H_{\tau}\left(x_{\tau}\right)=\left[\begin{array}{llll}
h_{\tau 1}\left(x_{\tau}\right) & \ldots & h_{\tau p}\left(x_{\tau}\right)
\end{array}\right]^{T}, \quad G(x)=\left[\begin{array}{ccc}
g_{11}(x) & \ldots & g_{1 p}(x) \\
\vdots & \ddots & \vdots \\
g_{p 1}(x) & \ldots & g_{p p}(x)
\end{array}\right] .
\end{aligned}
$$

Then, the system (1) can be rewritten in the following compact form:

$$
y^{(r)}=F(x)+G(x) \Phi(u)+H_{\tau}\left(x_{\tau}\right),
$$

where $F(),. H_{\tau}(),. \Phi(.) \in R^{p}$ and $G(.) \in R^{p \times p}$.

The objective of this paper is to design a stable control system allowing the system output $y$ to follow a specified desired trajectory $y_{d}=\left[y_{d 1}, \ldots, y_{d p}\right]^{T} \in R^{p}$ assuming that the vector $x_{d}=\left[y_{d 1}, \dot{y}_{d 1}, \ldots, y_{d 1}^{\left(r_{1}-1\right)}, y_{d 1}^{\left(r_{1}\right)}, \ldots, y_{d p}, \dot{y}_{d p}, \ldots\right.$, $\left.y_{d p}^{\left(r_{p}-1\right)}, y_{d p}^{\left(r_{p}\right)}\right]^{T}$ is continuous, bounded and available for measurement. Then $x_{d} \in \Omega_{x_{d}} \subset R^{r+p}$ with $\Omega_{x_{d}}$ is a known compact set.

Let us define the tracking error as

$$
\begin{gathered}
e_{1}=y_{1}-y_{d 1}, \\
\vdots \\
e_{p}=y_{p}-y_{d p},
\end{gathered}
$$


and the filtered tracking error as

$$
S=\left[S_{1}, \ldots, S_{p}\right]^{T},
$$

with

$$
S_{i}=\left[\frac{d}{d t}+\lambda_{i}\right]^{r_{i}-1} e_{i}, \quad \text { for } \lambda_{i}>0 \forall i=1, \ldots, p .
$$

Then, we can rewrite (5) as follows:

$$
S_{i}=\lambda_{i}^{r_{i}-1} e_{i}+\left(r_{i}-1\right) \lambda_{i}^{r_{i}-2} \dot{e}_{i}+\cdots+\left(r_{i}-1\right) \lambda_{i} e_{i}^{\left(r_{i}-2\right)}+e_{i}^{\left(r_{i}-1\right)},
$$

with $i=1, \ldots, p$.

Notice that if we choose $\lambda_{i}>0$, with $i=1, \ldots, p$, then the roots of the polynomial $H_{i}(s)=\lambda_{i}^{r_{i}-1}+\left(r_{i}-1\right) \lambda_{i}^{r_{i}-2}$ $s+\cdots+\left(r_{i}-1\right) \lambda_{i} s^{r_{i}-2}+s^{r_{i}-1}$ related to the characteristic equation of $S_{i}=0$ are all in the open left-half plane.

The relation (6) can be rewritten in the following compact form:

$$
S_{i}=C_{i}^{T} E_{i},
$$

with

$$
\begin{aligned}
& E_{i}=\left[\begin{array}{lllll}
e_{i} & \dot{e}_{i} & \ldots & e_{i}^{\left(r_{i}-2\right)} & e_{i}^{\left(r_{i}-1\right)}
\end{array}\right]^{T}, \\
& C_{i}^{T}=\left[\begin{array}{ll}
\lambda_{i}^{r_{i}-1} & \left(r_{i}-1\right) \lambda_{i}^{r_{i}-2} \ldots\left(r_{i}-1\right) \lambda_{i} 1
\end{array}\right] .
\end{aligned}
$$

Consequently, the vector $S$ takes the form

$$
S=C^{T} E,
$$

where

$$
\begin{aligned}
& C^{T}=\operatorname{diag}\left[\begin{array}{llll}
C_{1}^{T} & C_{2}^{T} & \ldots & C_{p}^{T}
\end{array}\right]_{(p \times r)}, \\
& E=\left[\begin{array}{llll}
E_{1}^{T} & E_{2}^{T} & \ldots & E_{p}^{T}
\end{array}\right]_{(r \times 1)}^{T} .
\end{aligned}
$$

And the dynamic of $S_{i}$ is described by

$$
\dot{S}_{i}=C_{r i}^{T} E_{i}+e_{i}^{\left(r_{i}\right)}, \quad i=1, \ldots, p,
$$

with

$$
C_{r i}^{T}=\left[\begin{array}{lllll}
0 & \lambda_{i}^{r_{i}-1} & \left(r_{i}-1\right) \lambda_{i}^{r_{i}-2} & \ldots & 0.5\left(r_{i}-1\right)\left(r_{i}-2\right) \lambda_{i}^{2}\left(r_{i}-1\right) \lambda_{i}
\end{array}\right] .
$$

The dynamic of $S$ can be written into the following compact form:

$$
\dot{S}=C_{r}^{T} E+e^{(r)},
$$

where

$$
\begin{aligned}
C_{r}^{T} & =\operatorname{diag}\left[\begin{array}{llll}
C_{r 1}^{T} & C_{r 2}^{T} & \ldots & C_{r p}^{T}
\end{array}\right]_{(p \times r)}, \\
e^{(r)} & =\left[\begin{array}{llll}
e_{1}^{\left(r_{1}\right)} & e_{2}^{\left(r_{2}\right)} & \ldots & e_{p}^{\left(r_{p}\right)}
\end{array}\right]^{T},
\end{aligned}
$$

with

$$
e^{(r)}=y^{(r)}-y_{d}^{(r)},
$$

where $y^{(r)}=\left[\begin{array}{llll}y_{1}^{\left(r_{1}\right)} & y_{2}^{\left(r_{2}\right)} & \ldots & y_{p}^{\left(r_{p}\right)}\end{array}\right]^{T}$ is previously defined, and

$$
y_{d}^{(r)}=\left[\begin{array}{llll}
y_{d 1}^{\left(r_{1}\right)} & y_{d 2}^{\left(r_{2}\right)} & \ldots & y_{d p}^{\left(r_{p}\right)}
\end{array}\right]^{T} .
$$

From (18), we can write (15) as follows:

$$
\dot{S}=C_{r}^{T} E+y^{(r)}-y_{d}^{(r)} .
$$

Thereafter (20) will be used in the development of the proposed controller and the stability analysis. 


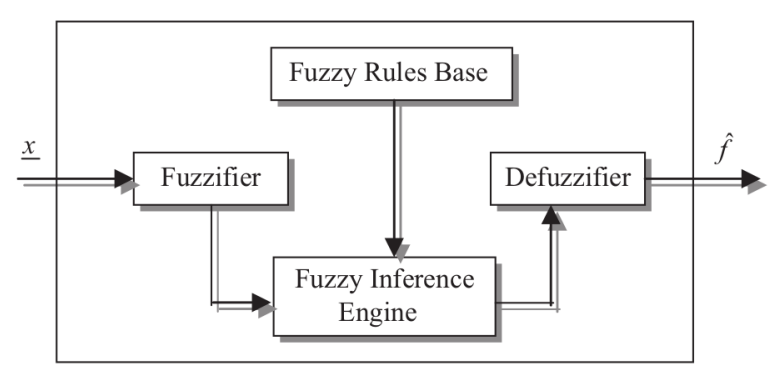

Fig. 1. The basic configuration of a fuzzy logic system.

\subsection{Description of the fuzzy logic system}

The basic configuration of a fuzzy logic system consists of a fuzzifier, some fuzzy IF-THEN rules, a fuzzy inference engine and a defuzzifier, as shown in Fig. 1. The fuzzy inference engine uses the fuzzy IF-THEN rules to perform a mapping from an input vector $\underline{x}^{T}=\left[x_{1}, x_{2}, \ldots, x_{n}\right] \in R^{n}$ to an output $\hat{f} \in R$. The ith fuzzy rule is written as

$$
R^{(i)}: \text { if } x_{1} \text { is } A_{1}^{i} \text { and } \ldots \text { and } x_{n} \text { is } A_{n}^{i} \text { then } \hat{f} \text { is } f^{i},
$$

where $A_{1}^{i}, A_{2}^{i}, \ldots$, and $A_{n}^{i}$ are fuzzy sets and $f^{i}$ is the fuzzy singleton for the output in the $i$ th rule. By using the singleton fuzzifier, product inference, and center-average defuzzifier, the output of the fuzzy system can be expressed as follows:

$$
\hat{f}(\underline{x})=\frac{\sum_{i=1}^{m} f^{i}\left(\prod_{j=1}^{n} \mu_{A_{j}^{i}}\left(x_{j}\right)\right)}{\sum_{i=1}^{m}\left(\prod_{j=1}^{n} \mu_{A_{j}^{i}}\left(x_{j}\right)\right)}=\theta^{T} \psi(\underline{x}),
$$

where $\mu_{A_{j}^{i}}\left(x_{j}\right)$ is the degree of membership of $x_{j}$ to $A_{j}^{i}, m$ is the number of fuzzy rules, $\theta^{T}=\left[f^{1}, f^{2}, \ldots, f^{m}\right]$ is the adjustable parameter vector (composed of consequent parameters), and $\psi^{T}=\left[\begin{array}{llll}\psi^{1} & \psi^{2} & \ldots & \psi^{m}\end{array}\right]$ with

$$
\psi^{i}(\underline{x})=\frac{\left(\prod_{j=1}^{n} \mu_{A_{j}^{i}}\left(x_{j}\right)\right)}{\sum_{i=1}^{m}\left(\prod_{j=1}^{n} \mu_{A_{j}^{i}}\left(x_{j}\right)\right)},
$$

being the fuzzy basis function (FBF). Throughout the paper, it is assumed that the FBFs are selected, so that there is always at least one active rule [33], i.e. $\sum_{i=1}^{m}\left(\prod_{j=1}^{n} \mu_{A_{j}^{i}}\left(x_{j}\right)\right)>0$.

It is worth noting that the fuzzy system (22) is commonly used in control applications. Following the universal approximation results, the fuzzy system (22) is able to approximate any nonlinear smooth function $f(\underline{x})$ on a compact operating space to an arbitrary degree of accuracy [33]. Of particular importance, it is assumed that the structure of the fuzzy system, namely the pertinent inputs, the number of membership functions for each input and the number of rules, and the membership function parameters are properly specified beforehand. The consequent parameters are then determined by appropriate parameter adaptation algorithms.

\subsection{Input nonlinearity model}

The mathematical model of the input nonlinearity (i.e. the sector nonlinearity and dead-zone) under consideration is described by

$$
\Phi_{i}\left(u_{i}\right)= \begin{cases}\phi_{i+}\left(u_{i}\right)\left(u_{i}-u_{i+}\right), & u_{i}>u_{i+}, \\ 0, & -u_{i-} \leq u_{i} \leq u_{i+}, \\ \phi_{i-}\left(u_{i}\right)\left(u_{i}+u_{i-}\right), & u_{i}<-u_{i-},\end{cases}
$$


where $\phi_{i+}\left(u_{i}\right)>0$ and $\phi_{i-}\left(u_{i}\right)>0$ are nonlinear functions of $u_{i}$, and $u_{i+}>0, u_{i_{-}}>0$ are known constants. The involved modelling assumption is

Assumption 1. The input nonlinearity $\Phi_{i}\left(u_{i}\right)$ satisfies the following important properties:

$$
\begin{array}{ll}
\left(u_{i}-u_{i+}\right) \Phi_{i}\left(u_{i}\right) \geq m_{i+}^{*}\left(u_{i}-u_{i+}\right)^{2}, & u_{i}>u_{i+}, \\
\left(u_{i}+u_{i-}\right) \Phi_{i}\left(u_{i}\right) \geq m_{i-}^{*}\left(u_{i}+u_{i-}\right)^{2}, & u_{i}<-u_{i-},
\end{array}
$$

where $m_{i+}^{*}$ and $m_{i-}^{*}$ are unknown constants which are called gain reduction tolerances.

Remark 1. It is worth mentioning that the models (24) and (25) allow to consider both dead-zones and sector nonlinearities with reduced prior knowledge. Indeed the gain reduction tolerances $m_{i+}^{*}$ and $m_{i-}^{*}$ are unknown, unlike $[17,23,25,26]$, and the input nonlinearity is only characterised throughout the property (25) together with the knowledge of the constants $u_{i+}$ and $u_{i-}$. By the way, notice that the dead zone considered in the contribution $[1,35]$ is a particular case of the above general form.

\subsection{Factorisation of the control-gain matrix}

Motivated by $[1,3,7,8,18,36]$, we need the following useful lemma in the control design.

Lemma 1 (Hsu et al. [18]). Any real matrix $G(x) \in R^{P \times P}$ with non-zero leading principal minors can be decomposed as follows:

$$
G(x)=G_{s}(x) D T(x),
$$

where $G_{S}(x) \in R^{P \times P}$ is a symmetric positive-definite matrix, $D \in R^{P \times P}$ is a diagonal matrix whose elements are +1 or -1 and $T(x) \in R^{P \times P}$ is a unity upper triangular matrix. The diagonal elements of $D$ are nothing than the ratios of the signs of the leading principal minors of $G(x)$.

\section{Proof of Lemma 1. See [18,27].}

It is worth noting that the decomposition (26) of the matrix $G(x)$ is very useful. The symmetric positive-definite matrix $G_{s}(x)$ will be exploited in the stability analysis. The unity upper triangular matrix $T(x)$ allows for algebraic loop free sequential determination of control components. The diagonal elements $d_{i i}$ of the matrix $D$ are nothing than the ratios of the signs of the leading principal minors of $G(x)$. This implies the following facts when the control-gain matrix has non-zero leading principal minors:

- if $G(x)$ is positive-definite, then $D=I_{P}$,

- if $G(x)$ is negative-definite, then $D=-I_{p}$, and

- if $G(x)$ is indefinite, the diagonal elements of the matrix $D$ are +1 and -1 .

\section{Design of the fuzzy adaptive controller}

In this section, we will develop a fuzzy adaptive variable-structure controller for the system (2) of which the controlgain matrix can be non-symmetric or even symmetric.

Using the matrix decomposition (26) and Eq. (20), the dynamics of $S$ can be rewritten as follows:

$$
G_{s}^{-1}(x) \dot{S}=G_{s}^{-1}(x)[v+F(x)]+D T(x) \Phi(u)+G_{s}^{-1}(x) H_{\tau}\left(x_{\tau}\right),
$$

where $v=C_{r}^{T} E-y_{d}^{(r)}$.

The following assumption is made on the control-gain matrix $G(x)$. 


\section{Assumption 2.}

(a) The matrix $G(x)$ has non-zero leading principal minors with known signs.

(b) $\partial g_{i j}(x) / \partial y_{i}^{\left(r_{i}-1\right)}=0, \forall i=1,2, \ldots, p$, and $j=1,2, \ldots, p$.

(c) $G_{s}(x)$ is of class $C^{1}$.

\section{Remark 2.}

(a) Assumption 2 is satisfied by many physical systems without time-delays such as robotic systems, induction motors, mechanical systems and chaotic systems [3].

(b) The required property on the leading principal minors (i.e. the leading principal minors of $G(x)$ must be non-zero) guarantees the existence of the matrix decomposition (26) and the controllability of the system (1).

(c) The required assumption on the partial derivatives of the control-gain matrix (i.e. Assumption (2b)) ensures that $d\left(D^{-1} G_{s}^{-1}(x) D\right) / d t$ depends only on the state vector $x$ (i.e. it ensures that $d\left(D^{-1} G_{s}^{-1}(x) D\right) / d t$ does not depend on the control input vector $u$ ).

Letting $G_{s 1}(x)=G_{s}^{-1}(x)$ and $F_{1}(x, u)=G_{s}^{-1}(x)[v+F(x)]+[D T(x)-D] \Phi(u)$, Eq. (27) becomes

$$
G_{S 1}(x) \dot{S}=F_{1}(x, u)+D \Phi(u)+G_{s 1}(x) H_{\tau}\left(x_{\tau}\right) .
$$

Now, let introduce the following change of the variables for stability analysis and control design simplicity

$$
\bar{S}=D^{-1} S,
$$

or $\bar{S}_{i}=d_{i i} S_{i}$ as $D=D^{T}=D^{-1}$ and $d_{i i}=+1$ or -1 .

This allows to rewrite (28) under the form

$$
G_{s 2}(x) \dot{\bar{S}}=D^{-1} F_{1}(x, u)+\Phi(u)+D^{-1} G_{s 1}(x) H_{\tau}\left(x_{\tau}\right),
$$

where $G_{s 2}(x)=D^{-1} G_{s 1}(x) D$. Due to the special forms of the matrices $D$ and $G_{s 1}(x), G_{s 2}(x)$ preserves the important properties of the original matrix $G_{s 1}(x)$ (or $G_{s}(x)$ ). Indeed, we can easily show that $G_{s 2}(x)$ is also symmetric and positive-definite. This property is of fundamental interest when investigating the control system stability.

We can rewrite (30) as follows:

$$
G_{s 2}(x) \dot{\bar{S}}+\frac{1}{2} \dot{G}_{s 2} \bar{S}=\alpha(\bar{z})+\Phi(u)+D^{-1} G_{s 1}(x) H_{\tau}\left(x_{\tau}\right)
$$

where $\alpha(\bar{z})=\left[\alpha_{1}\left(\bar{z}_{1}\right), \alpha_{2}\left(\bar{z}_{2}\right), \ldots, \alpha_{p}\left(\bar{z}_{p}\right)\right]^{T}=D^{-1} F_{1}(x, u)+\frac{1}{2} \dot{G}_{s 2}(x) \bar{S}$, with $\bar{z}=\left[\bar{z}_{1}^{T}, \bar{z}_{2}^{T}, \ldots, \bar{z}_{p}^{T}\right]^{T}$.

By carefully examining the expressions of $F_{1}(x, u)$ and $\alpha(\bar{z})$, the elements of the vector $\bar{z}$ can be selected as follows:

$$
\begin{gathered}
\bar{z}_{1}=\left[x^{T}, S^{T}, u_{2}, \ldots, u_{p}\right]^{T}, \\
\bar{z}_{2}=\left[x^{T}, S^{T}, u_{3}, \ldots, u_{p}\right]^{T}, \\
\vdots \\
\bar{z}_{p-1}=\left[x^{T}, S^{T}, u_{p}\right]^{T}, \\
\bar{z}_{p}=\left[x^{T}, S^{T}\right]^{T} .
\end{gathered}
$$

It is clear from the propriety of the matrix $D T(x)-D$ that $\bar{z}_{1}$ depends on control inputs $u_{2}, \ldots, u_{p}, \bar{z}_{2}$ depends on $u_{3}, \ldots, u_{p}$ and so on. The nonlinearity $\alpha(\bar{z})$ has an upper triangular control structure, allowing thereby for algebraic loop free sequential determination of the control variables.

Let us define the following compact sets:

$$
\Omega_{\bar{z}_{i}}=\left\{\left[x^{T}, S^{T}, u_{i+1}, \ldots, u_{p}\right]^{T} \mid x \in \Omega_{x} \subset R^{r}, x_{d} \in \Omega_{x_{d}}\right\}, \quad i=1,2, \ldots, p-1,
$$

and

$$
\Omega_{\bar{z}_{p}}=\left\{\left[x^{T}, S^{T}\right] \mid x \in \Omega_{x} \subset R^{r}, x_{d} \in \Omega_{x_{d}}\right\} .
$$


Posing $D^{-1} G_{s 1}(x) H_{\tau}\left(x_{\tau}\right)=\bar{H}_{\tau}\left(x, x_{\tau}\right)=\left[\bar{h}_{\tau 1}\left(x, x_{\tau}\right), \ldots, \bar{h}_{\tau p}\left(x, x_{\tau}\right)\right]^{T}$, Eq. (31) becomes

$$
\bar{S}^{T} G_{s 2}(x) \dot{\bar{S}}+\frac{1}{2} \bar{S}^{T} \dot{G}_{s 2} \bar{S}=\bar{S}^{T} \alpha(\bar{z})+\bar{S}^{T} \Phi(u)+\sum_{i=1}^{p} \bar{S}_{i} \bar{h}_{\tau i}\left(x, x_{\tau}\right)
$$

Now let us denote

$$
\begin{aligned}
& \bar{E}=\left[\bar{E}_{1}^{T}, \ldots, \bar{E}_{p}^{T}\right]^{T} \text { with } \bar{E}_{i}=\left[e_{i}, \dot{e}_{i}, \ldots, e_{i}^{\left(r_{i}-2\right)}\right]^{T}=\left[e_{i 1}, e_{i 2}, \ldots, e_{i, r_{i}-1}\right]^{T} \text { for } i=1, \ldots, p, \\
& \bar{E}_{\tau}=\left[\bar{E}_{\tau 1}^{T}, \ldots, \bar{E}_{\tau p}^{T}\right]^{T} \text { with } \bar{E}_{\tau i}=\left[e_{i 1}\left(t-\tau_{i}(t)\right), e_{i 2}\left(t-\tau_{i}(t)\right), \ldots, e_{i, r_{i}-1}\left(t-\tau_{i}(t)\right)\right]^{T} \text { for } i=1, \ldots, p, \\
& x_{d \tau}=\left[x_{d \tau 1}^{T}, \ldots, x_{d \tau p}^{T}\right]^{T}, x_{d \tau \mathrm{i}}=\left[y_{d i}\left(t-\tau_{i}(t)\right), \dot{y}_{d i}\left(t-\tau_{i}(t)\right), \ldots, y_{d i}^{\left(r_{i}-1\right)}\left(t-\tau_{i}(t)\right)\right]^{T} \text { for } i=1, \ldots, p .
\end{aligned}
$$

Eq. (33) can be expressed as follows:

$$
\bar{S}^{T} G_{s 2}(x) \dot{\bar{S}}+\frac{1}{2} \bar{S}^{T} \dot{G}_{s 2} \bar{S}=\bar{S}^{T} \alpha(\bar{z})+\bar{S}^{T} \Phi(u)+\sum_{i=1}^{p} \bar{S}_{i}\left(\bar{h}_{\tau i}\left(x, x_{\tau}\right)-\bar{h}_{\tau i}\left(x, x_{d \tau}\right)\right)+\sum_{i=1}^{p} \bar{S}_{i} \bar{h}_{\tau i}\left(x, x_{d \tau}\right) .
$$

The following realistic assumptions are made.

Assumption 3. The unknown nonlinear function $\bar{h}_{\tau i}\left(x_{\tau}\right)$ satisfies

$$
\left|\bar{h}_{\tau i}\left(x, x_{\tau}\right)-\bar{h}_{\tau i}\left(x, x_{d \tau}\right)\right| \leq \bar{k}_{1 i}\left\|\bar{E}_{\tau}\right\|+\bar{k}_{0 i}
$$

where $\bar{k}_{0 i}$ and $\bar{k}_{1 i}$ are unknown positive constants.

Assumption 4. There exists an unknown continuous positive function $\bar{\alpha}_{i}\left(\bar{z}_{i}\right)$ such that

$$
\left|\alpha_{i}\left(\bar{z}_{i}\right)+\bar{h}_{\tau i}\left(x, x_{d \tau}\right)\right| \leq \eta \bar{\alpha}_{i}\left(\bar{z}_{i}\right), \quad \forall \bar{z}_{i} \in \Omega_{\bar{z}_{i}},
$$

with $\eta=\min _{i}\left\{\eta_{i}\right\}$, where $\eta_{i}=\min \left\{m_{i+}^{*}, m_{i-}^{*}\right\}$.

Assumption 5. The time-varying delays $\tau_{i}(t)$ satisfy the following relations:

$$
0 \leq \tau_{i}(t) \leq \tau_{i}^{*}, \quad \dot{\tau}_{i}(t) \leq \bar{\tau}_{i}<1, \forall i=1, \ldots, p,
$$

where $\tau_{i}^{*}$ and $\bar{\tau}_{i}$ are unknown constants.

\section{Remark 3.}

(a) It is worth mentioning that the notations used above Eq. (34) are only valid for $r_{i} \geq 2$. In the case where $r_{i}=1$, the vectors $\bar{E}_{i}$ and $\bar{E}_{\tau i}$ become as follows: $\bar{E}_{i}=e_{i 1}=e_{i}$ and $\bar{E}_{\tau i}=e_{i 1}\left(t-\tau_{i}(t)\right)$.

(b) Assumption 3 is not restrictive. It is generally used in the literature.

Remark 4. Note that the continuous function $h_{\tau i}\left(x_{d \tau}\right)$ is bounded since the signal $x_{d \tau}$ is bounded. Hence, Assumption 4 is by no means restrictive since such function $\bar{\alpha}_{i}\left(\bar{z}_{i}\right)$ always exists.

Remark 5. The choice of the vectors $\bar{z}_{i}$ (input arguments of the unknown functions $\bar{\alpha}_{i}$ ) is not unique. In fact, since we know that $S$ and $u$ are functions of state $x$ and $x_{d}$, then it can be seen quite simple that all $\bar{z}_{i}$ are functions of $x$ and $x_{d}$ (e.g. we can choose $\bar{z}_{i}=\left[x^{T}, x_{d}^{T}\right]^{T}$ or $\bar{z}_{i}=\left[x^{T}, E^{T}\right]^{T}$ with $i=1,2, \ldots, p$ ). Also, since $x_{d}$ is bounded, we can choose $\bar{z}_{i}=x$. 
From the relation (6), we can easily show that the dynamics of $\bar{E}_{i}$ are described by

$$
\dot{\bar{E}}_{i}=A_{c i} \bar{E}_{i}+B_{i} S_{i}, \quad \forall i=1, \ldots, 2 .
$$

where

$$
\begin{aligned}
A_{c i} & =\left[\begin{array}{ccccc}
0 & 1 & 0 & \cdots & 0 \\
0 & 0 & 1 & \cdots & 0 \\
\vdots & \vdots & \vdots & \ddots & \vdots \\
0 & 0 & 0 & \cdots & 1 \\
-\lambda_{i}^{r_{i}-1} & -\left(r_{i}-1\right) \lambda_{i}^{r_{i}-2} & -\frac{1}{2}\left(r_{i}-1\right)\left(r_{i}-2\right) \lambda_{i}^{r_{i}-3} & \cdots & -\left(r_{i}-1\right) \lambda_{i}
\end{array}\right] \in R^{\left(r_{i}-1\right) \times\left(r_{i}-1\right)}, \\
B_{i} & =\left[\begin{array}{c}
0 \\
\vdots \\
0 \\
1
\end{array}\right] \in R^{\left(r_{i}-1\right)} .
\end{aligned}
$$

Since the matrix $A_{c i}$ is stable (i.e. Hurwitz), then for any matrix given $Q_{i}=Q_{i}^{T}>0$, there exists a single matrix $P_{i}=P_{i}^{T}>0$ which is the solution of the Lyapunov equation:

$$
P_{i} A_{c i}+A_{c i}^{T} P_{i}=-Q_{i}
$$

It is worth denoting that Eqs. (37) and (38) will be used later in the stability analysis.

The unknown continuous function $\bar{\alpha}_{i}\left(\bar{z}_{i}\right)$ can be approximated by the fuzzy system (22) as follows:

$$
\hat{\bar{\alpha}}_{i}\left(\bar{z}_{i}, \theta\right)=\theta_{i}^{T} \psi_{i}\left(\bar{z}_{i}\right),
$$

where $\psi_{i}\left(\bar{z}_{i}\right)$ is the FBF vector, which is fixed a priori by the designer, and $\theta_{i}$ is the adjustable parameter vector of the fuzzy system.

Following the universal approximation theorem [33], $\bar{\alpha}_{i}\left(\bar{z}_{i}\right)$ is approximated by an ideal fuzzy system as follows:

$$
\bar{\alpha}_{i}\left(\bar{z}_{i}\right)=\hat{\bar{\alpha}}_{i}\left(\bar{z}_{i}, \theta_{i}^{*}\right)+\varepsilon_{i}\left(\bar{z}_{i}\right)=\theta_{i}^{* T} \psi_{i}\left(\bar{z}_{i}\right)+\varepsilon_{i}\left(\bar{z}_{i}\right),
$$

where $\theta_{i}^{*}$ are optimal values of $\theta_{i}$. They are assumed to be constant and unknown.

As in the literature [1-6,9,13,21,22,24,29-31], the fuzzy approximation error is assumed to be bounded as follows:

$$
\left|\varepsilon_{i}\left(\bar{z}_{i}\right)\right| \leq \bar{\varepsilon}_{i}, \quad \forall \bar{z}_{i} \in \Omega_{\bar{z}_{i}},
$$

where $\bar{\varepsilon}_{i}$ is an unknown constant.

From the above analysis, we have

$$
\begin{aligned}
\hat{\bar{\alpha}}_{i}\left(\bar{z}_{i}, \theta_{i}\right)-\bar{\alpha}_{i}\left(\bar{z}_{i}\right) & =\hat{\bar{\alpha}}_{i}\left(\bar{z}_{i}, \theta_{i}\right)-\hat{\bar{\alpha}}_{i}\left(\bar{z}_{i}, \theta_{i}^{*}\right)+\hat{\bar{\alpha}}_{i}\left(\bar{z}_{i}, \theta_{i}^{*}\right)-\bar{\alpha}_{i}\left(\bar{z}_{i}\right) \\
& =\hat{\bar{\alpha}}_{i}\left(\bar{z}_{i}, \theta_{i}\right)-\hat{\bar{\alpha}}_{i}\left(\bar{z}_{i}, \theta_{i}^{*}\right)-\varepsilon_{i}\left(\bar{z}_{i}\right)=\tilde{\theta}_{i}^{T} \psi_{i}\left(\bar{z}_{i}\right)-\varepsilon_{i}\left(\bar{z}_{i}\right) .
\end{aligned}
$$

The following fuzzy adaptive variable-structure controller is proposed to perform the required tracking control objective.

$$
u_{i}= \begin{cases}-\rho_{i}(t) \operatorname{sign}\left(\bar{S}_{i}\right)-u_{i-}, & \bar{S}_{i}>0, \\ 0, & \bar{S}_{i}=0, \\ -\rho_{i}(t) \operatorname{sign}\left(\bar{S}_{i}\right)+u_{i+}, & \bar{S}_{i}<0,\end{cases}
$$


with $\rho_{i}(t)=k_{0 i}+\left(k_{1 i}+k_{2 i}\right)\left|\bar{S}_{i}\right|+\theta_{i}^{T} \psi_{i}\left(\bar{z}_{i}\right), \forall i=1, \ldots, p$ and

$$
\begin{aligned}
& \dot{k}_{0 i}=-\gamma_{0 i} \sigma_{0 i} k_{0 i}+\gamma_{0 i}\left|\bar{S}_{i}\right|, \quad k_{0 i}(0)>0, \\
& \dot{k}_{1 i}=-\gamma_{1 i} \sigma_{1 i} k_{1 i}+\gamma_{1 i} \bar{S}_{i}^{2}, \quad k_{1 i}(0)>0, \\
& \dot{\theta}_{i}=-\gamma_{2 i} \sigma_{2 i} \theta_{i}+\gamma_{2 i}\left|\bar{S}_{i}\right| \psi_{i}\left(\bar{z}_{i}\right), \quad \theta_{i j}(0)>0,
\end{aligned}
$$

where $\gamma_{0 i}, \gamma_{1 i}, \gamma_{2 i}, \sigma_{0 i}, \sigma_{1 i}, \sigma_{2 i}, k_{2 i}>0$ are design constants, $k_{0 i}, k_{1 i}$ and $\theta_{i}$ are the online estimates of the uncertain terms $k_{0 i}^{*}=\bar{\varepsilon}_{i}+\bar{k}_{0 i} / \eta, k_{1 i}^{*}=\left(\bar{k}_{1 i}\right)^{2} / 2 \eta l+\left\|P_{i} B_{i}\right\|^{2} / \eta$ and $\theta_{i}^{*}$, respectively. Note that $l$ is an unknown positive constant given by

$$
l=\frac{1}{\max _{i}\left\{\frac{e^{\omega \tau_{i}^{*}}}{1-\bar{\tau}_{i}}\right\}},
$$

with $\omega$ is a positive constant which will be later specified in Appendix A.

Remark 6. With $k_{0 i}(0)>0, k_{1 i}(0)>0$ and $\theta_{i}(0)>0$, it follows from adaptive laws (43)-(45) that their respective solutions satisfy $k_{0 i}(t)>0, k_{1 i}(t)>0$ and $\theta_{i}(t)>0$ for $t>0$.

Using Assumptions 3 and 4 and Eq. (34), and the fact

$$
\sum_{i=1}^{p} \bar{k}_{1 i}\left|\bar{S}_{i}\right|\left\|\bar{E}_{\tau}\right\| \leq \frac{1}{2 l} \sum_{i=1}^{p} \bar{k}_{1 i}^{2} \bar{S}_{i}^{2}+\frac{l p}{2}\left\|\bar{E}_{\tau}\right\|^{2},
$$

we have

$$
\begin{aligned}
\frac{1}{\eta} \bar{S}^{T} G_{s 2}(x) \dot{\bar{S}}+\frac{1}{2 \eta} \bar{S}^{T} \dot{G}_{s 2} \bar{S} & \leq \sum_{i=1}^{p}\left|\bar{S}_{i}\right| \bar{\alpha}_{i}\left(\bar{z}_{i}\right)+\frac{1}{\eta} \bar{S}^{T} \Phi(u)+\frac{1}{\eta} \sum_{i=1}^{p} \bar{S}_{i}\left(\bar{h}_{\tau i}\left(x, x_{\tau}\right)-\bar{h}_{\tau i}\left(x, x_{d \tau}\right)\right) \\
& \leq \sum_{i=1}^{p}\left|\bar{S}_{i}\right| \bar{\alpha}_{i}\left(\bar{z}_{i}\right)+\frac{1}{\eta} \bar{S}^{T} \Phi(u)+\frac{1}{\eta} \sum_{i=1}^{p} \bar{k}_{1 i}\left|\bar{S}_{i}\right|\left\|\bar{E}_{\tau}\right\|+\frac{1}{\eta} \sum_{i=1}^{p} \bar{k}_{0 i}\left|\bar{S}_{i}\right| \\
& \leq \sum_{i=1}^{p}\left|\bar{S}_{i}\right| \bar{\alpha}_{i}\left(\bar{z}_{i}\right)+\frac{1}{\eta} \bar{S}^{T} \Phi(u)+\frac{1}{2 \eta l} \sum_{i=1}^{p} \bar{k}_{1 i}^{2} \bar{S}_{i}^{2}+\frac{l p}{2 \eta}\left\|\bar{E}_{\tau}\right\|^{2}+\frac{1}{\eta} \sum_{i=1}^{p} \bar{k}_{0 i}\left|\bar{S}_{i}\right| .
\end{aligned}
$$

From (41) and (46), we get

$$
\begin{aligned}
\frac{1}{\eta} \bar{S}^{T} G_{s 2}(x) \dot{\bar{S}}+\frac{1}{2 \eta} \bar{S}^{T} \dot{G}_{s 2} \bar{S} \leq & -\sum_{i=1}^{p}\left|\bar{S}_{i}\right|\left(\tilde{k}_{0 i}+\tilde{k}_{1 i}\left|\bar{S}_{i}\right|+\tilde{\theta}_{i}^{T} \psi_{i}\left(\bar{z}_{i}\right)\right)+\sum_{i=1}^{p}\left|\bar{S}_{i}\right|\left(k_{0 i}+k_{1 i}\left|\bar{S}_{i}\right|+\theta_{i}^{T} \psi_{i}\left(\bar{z}_{i}\right)\right) \\
& +\frac{1}{\eta} \bar{S}^{T} \Phi(u)-\sum_{i=1}^{p} \frac{\left\|P_{i} B_{i}\right\|^{2}}{\eta} \bar{S}_{i}^{2}+\frac{l p}{2 \eta}\left\|\bar{E}_{\tau}\right\|^{2}
\end{aligned}
$$

where

$$
\begin{aligned}
& \tilde{k}_{0 i}=k_{0 i}-k_{0 i}^{*}=k_{0 i}-\bar{\varepsilon}_{i}-\left(\bar{k}_{0 i} / \eta\right), \\
& \tilde{k}_{1 i}=k_{1 i}-k_{1 i}^{*}=k_{1 i}-\left(\bar{k}_{1 i}^{2} / 2 \eta l\right)-\left(\left\|P_{i} B_{i}\right\|^{2} / \eta\right), \text { and } \\
& \tilde{\theta}_{i}=\theta_{i}-\theta_{i}^{*} .
\end{aligned}
$$

Theorem 1. Consider the system (2) and suppose that Assumptions 1-5 are satisfied. Then, the control law defined by (42)-(45) guarantees the following properties:

- All signals in the closed-loop system are uniformly ultimately bounded.

- The solution of the closed-loop error system exponentially converges to an adjustable region.

Proof. Given in Appendix A. 


\section{Remark 7.}

(1) In the case where $u_{i+}=u_{i-}=u_{i 0}$, the expression (42) can be simplified as follows:

$$
u_{i}=-\left(\rho_{i}(t)+u_{i 0}\right) \operatorname{sign}\left(\bar{S}_{i}\right),
$$

with $\rho_{i}(t)=k_{0 i}+\left(k_{1 i}+k_{2 i}\right)\left|\bar{S}_{i}\right|+\theta_{i}^{T} \psi_{i}\left(\bar{z}_{i}\right)$.

(2) The sign function in (42) and (48) has to be replaced by any equivalent smooth function to deal with the chattering effects.

Remark 8. It should be noted that the fuzzy system (22) (used here for approximating the unknown nonlinear functions) can be replaced by any linearly parameterized networks without any technical difficulty such as RBF, polynomial, splines and wavelet networks. However, only the fuzzy systems, by design, provide a systematic and efficient framework to incorporate the linguistic fuzzy information from human experts.

\section{Simulation results}

Simulation studies are carried out to show the effectiveness of the proposed controller. Two control problems are considered to this end. The first one concerns an academic MIMO system with time-varying delays in states and actuator nonlinearities in the input, while the second one concerns a two-link rigid robot manipulator subject to actuator nonlinearities.

\subsection{Example 1}

In the following, we present simulation results showing the performances of the proposed fuzzy adaptive controller applied to an academic MIMO nonlinear time-delay system subject to actuator nonlinearities and having a nonsymmetric indefinite control-gain matrix. The dynamics of this MIMO system are given by

$$
\left\{\begin{aligned}
\dot{x}_{11}= & x_{12}, \\
\dot{x}_{12}= & x_{21}-0.3 \sin \left(x_{11} x_{12}\right)+x_{12}^{2}+\left(2+\cos \left(x_{11}\right)\right) \Phi_{1}\left(u_{1}\right)+\left(1+\left(\sin \left(x_{21}\right)\right)^{2}\right) \Phi_{2}\left(u_{2}\right) \\
& +0.9 x_{11}\left(t-\tau_{1}(t)\right) \sin \left(x_{11}\left(t-\tau_{1}(t)\right),\right. \\
\dot{x}_{21}= & x_{22}, \\
\dot{x}_{22}= & x_{22}^{3}+e^{x_{11}}-1+x_{12}^{2}-0.5 \Phi_{1}\left(u_{1}\right)-\left(1+\left(\sin \left(x_{21}\right)\right)^{2}\right) \Phi_{2}\left(u_{2}\right)+1.3 x_{21}\left(t-\tau_{2}(t)\right), \\
y_{1}= & x_{11}, y_{2}=x_{21} .
\end{aligned}\right.
$$

Let $y=\left[y_{1}, y_{2}\right]^{T}, u=\left[u_{1}, u_{2}\right]^{T}, \Phi(u)=\left[\Phi_{1}\left(u_{1}\right), \Phi_{2}\left(u_{2}\right)\right]^{T}$, and $x=\left[x_{11}, x_{12}, x_{21}, x_{22}\right]^{T}$. Then, the system (49) can be given in the following form:

$$
\ddot{y}=F(x)+G(x) \Phi(u)+H_{\tau}\left(x_{\tau}\right),
$$

where

$$
\begin{aligned}
& F(x)=\left(\begin{array}{c}
x_{21}-0.3 \sin \left(x_{11} x_{12}\right)+x_{12}^{2} \\
x_{22}^{3}+e^{x_{11}}-1+x_{12}^{2}
\end{array}\right), \quad G(x)=\left(\begin{array}{cc}
\left(2+\cos \left(x_{11}\right)\right) & 1+\left(\sin \left(x_{21}\right)\right)^{2} \\
-0.5 & -\left(1+\left(\sin \left(x_{21}\right)\right)^{2}\right)
\end{array}\right), \\
& H_{\tau}\left(x_{\tau}\right)=\left(\begin{array}{c}
0.9 x_{11}\left(t-\tau_{1}(t)\right) \sin \left(x_{11}\left(t-\tau_{1}(t)\right)\right) \\
1.3 x_{21}\left(t-\tau_{2}(t)\right)
\end{array}\right) .
\end{aligned}
$$

The input nonlinearities $\Phi_{i}\left(u_{i}\right)$ for $i=1,2$ are described by

$$
\Phi_{i}\left(u_{i}\right)= \begin{cases}\left(u_{i}-3\right)\left(1-0.3 \sin \left(u_{i}\right)\right), & u_{i}>3, \\ 0, & -3 \leq u_{i} \leq 3, \\ \left(u_{i}+3\right)\left(0.8-0.3 \cos \left(u_{i}\right)\right), & u_{i}<-3 .\end{cases}
$$


It is worth noting that the matrix $G(x)$ is non-symmetric and indefinite. Recall that the system nonlinearities, i.e. $F(x)$, $H_{\tau}\left(x_{\tau}\right), G(x)$, and $\Phi(u)$, are assumed to be unknown except the signs of the leading principal minors of $G(x)$, the property (25) of $\Phi(u)$ and constants $u_{i+}$ and $u_{i-}$.

The system (50) is very complicated and many reasons make its control a challenge, namely:

- The numerical example (50) is a MIMO nonlinear unknown system with a non-symmetric indefinite control-gain matrix. Note that in all previous fuzzy adaptive control schemes, the matrix $G(x)$ has been assumed to be positivedefinite or negative-definite.

- It contains unknown nonlinear functions with time-varying delays.

- It is subject to unavoidable actuator nonlinearities (i.e. sector nonlinearities and dead-zone).

The control objective is to force the system outputs $y_{1}$ and $y_{2}$ to track the sinusoidal desired trajectories $y_{d 1}=\sin (t)$ and $y_{d 2}=\sin (t)$, respectively.

The fuzzy system $\theta_{1}^{T} \psi_{1}\left(\bar{z}_{1}\right)$ has the vector $\left[x^{T}, u_{2}\right]^{T}$ as input, while the fuzzy system $\theta_{2}^{T} \psi_{2}\left(\bar{z}_{2}\right)$ has the state vector $x$ as input. For each variable of the entries of these fuzzy systems, as in [2], we define three (one triangular and two trapezoidal) membership functions uniformly distributed on the intervals $[-2,2]$ for $x_{11}, x_{12}, x_{21}$, and $x_{22}$, and $[-25,25]$ for $u_{2}$. The design parameters used in this simulation are chosen as follows: $\gamma_{01}=\gamma_{02}=20, \gamma_{11}=20$, $\gamma_{12}=20, \gamma_{21}=1500, \gamma_{22}=1500, \sigma_{01}=\sigma_{02}=0.01, \sigma_{11}=\sigma_{12}=0.01, \sigma_{21}=0.001, \sigma_{22}=0.001, \lambda_{1}=$ $\lambda_{2}=5, k_{21}=k_{22}=2$. The initial conditions are selected as: $x(0)=\left[\begin{array}{llll}1 & 0 & 1 & 0\end{array}\right], k_{01}(0)=k_{02}(0)=0.01, k_{11}(0)=$ $k_{12}(0)=0.01, \theta_{1 i}(0)=\theta_{2 i}(0)=0.001$. Time-varying delays are chosen as follows: $\tau_{1}(t)=0.2(1+\sin (t)), \tau_{2}(t)=$ $1-0.5 \cos (t)$.

The following cases are simulated to illustrate the validity of the proposed controller:

- The discontinuous controller (48) is applied to the system (50).

- A practical (smooth) version of the controller (48) is applied to the system (50).

(a) Simulation results with the discontinuous function $\operatorname{sign}\left(\bar{S}_{i}\right)$ : Fig. 2 shows the simulation results of the proposed controller. Fig. 2(a) and (b) shows that the tracking errors are bounded and converge towards small values. Fig. 2(c) illustrates the boundedness of the control signals $u_{i}$. Fig. 2(d) shows the boundedness of the norm of the adaptive fuzzy parameters, i.e. $\left\|\theta_{1}\right\|=\sqrt{\theta_{1}^{T} \theta_{1}}$ and $\left\|\theta_{2}\right\|=\sqrt{\theta_{2}^{T} \theta_{2}}$.

In Fig. 3(a) and (b), phase planes of $\left(e_{1}, \dot{e}_{1}\right)$ and $\left(e_{2}, \dot{e}_{2}\right)$ are, respectively, depicted. These figures show that for both subsystems after a relatively fast reaching mode, a sliding mode is enforced and is maintained by producing a suitable control signal. Fig. 3(c) and (d) illustrates the filtered tracking errors $S_{1}$ and $S_{2}$, respectively. From these results, it is clear that the filtered tracking errors converge in the vicinity of the origin.

In summary, in spite of the presence of the input nonlinearities, the unknown time-varying delays in states and uncertain dynamics, the MIMO system (50) remains stable and with a weak-tracking error. Nevertheless, from these results, we can see clearly the chattering phenomenon and the non-smoothness of the control signals.

(b) Simulation results with the smooth function $\tanh \left(k_{s i} \bar{S}_{i}\right)$ : In order to eliminate the chattering effect, the discontinuous function $\operatorname{sign}\left(\bar{S}_{i}\right)$ in (48) is replaced here by an equivalent smooth function: $\tanh \left(k_{s i} \bar{S}_{i}\right)$ with $k_{s i}=10$.

The obtained results are shown in Figs. 4 and 5. From these figures, we can see that the system tracks its desired trajectories and the control inputs are smooth.

\subsection{Example 2}

In this section, we present simulation results showing the tracking performances of the proposed controller applied to a two-link rigid robot manipulator which moves in a horizontal plane. The dynamics of this MIMO system are given by $[3,21]$

$$
\left(\begin{array}{l}
\ddot{q}_{1} \\
\ddot{q}_{2}
\end{array}\right)=\left(\begin{array}{ll}
M_{11} & M_{12} \\
M_{21} & M_{22}
\end{array}\right)^{-1}\left\{\left(\begin{array}{l}
\Phi_{1}\left(u_{1}\right) \\
\Phi_{2}\left(u_{2}\right)
\end{array}\right)-\left(\begin{array}{cc}
-h \dot{q}_{2} & -h\left(\dot{q}_{1}+\dot{q}_{2}\right) \\
h \dot{q}_{1} & 0
\end{array}\right)\left(\begin{array}{l}
\dot{q}_{1} \\
\dot{q}_{2}
\end{array}\right)\right\},
$$



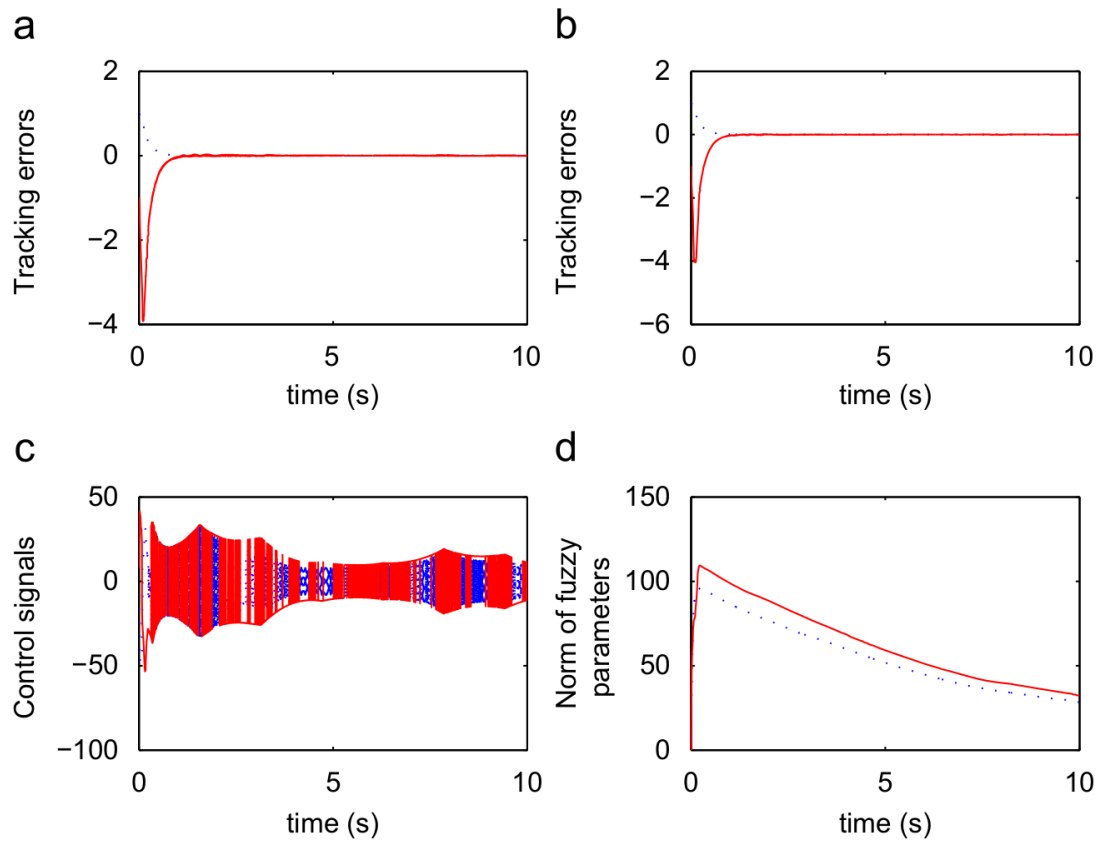

Fig. 2. Simulation results for example 1 (case 1): (a) Tracking errors: $e_{1}$ (dotted line) and $\dot{e}_{1}$ (solid line). (b) Tracking errors: $e_{2}$ (dotted line) and $\dot{e}_{2}$ (solid line). (c) Control input signals: $u_{1}$ (dotted line) and $u_{2}$ (solid line). (d) Norm of fuzzy parameters: $\left\|\theta_{1}\right\|$ (dotted line), $\left\|\theta_{2}\right\|$ (solid line).

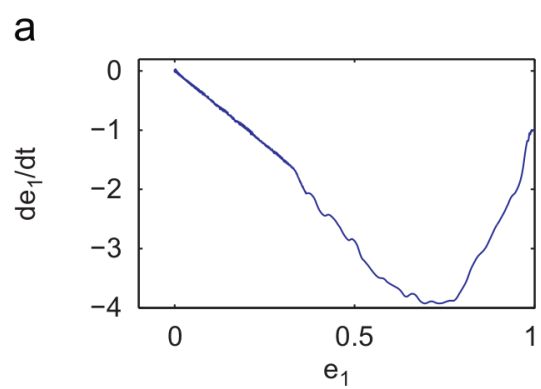

b
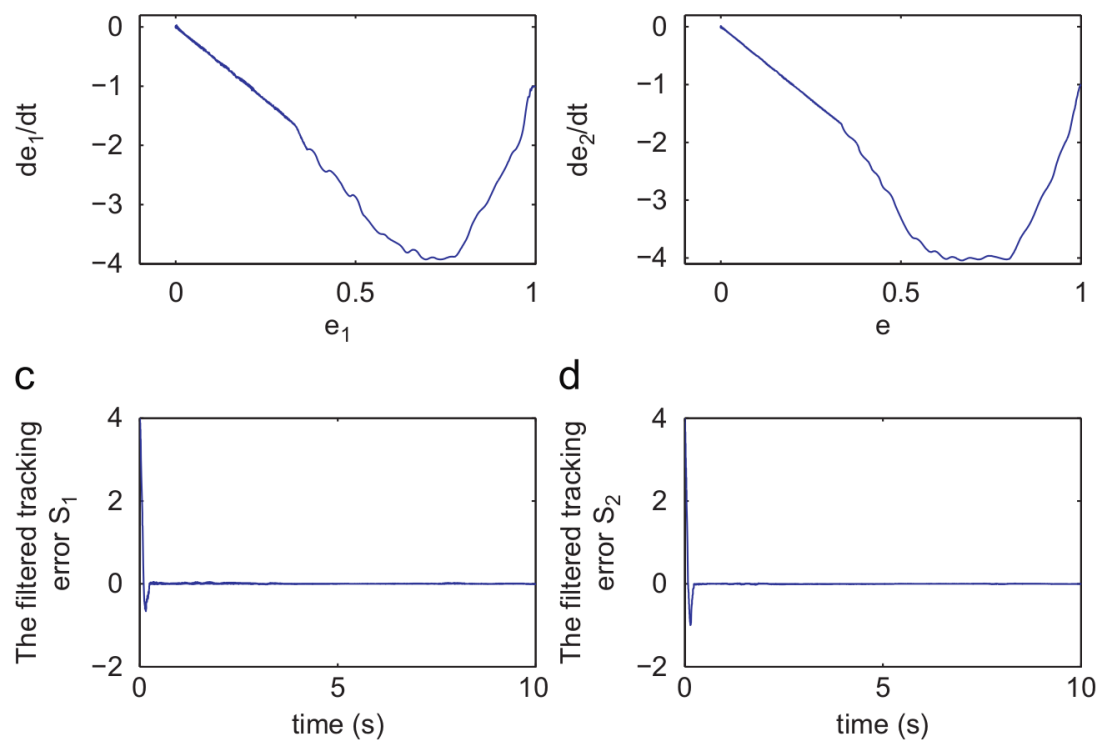

Fig. 3. Simulation results for example 1 (case 1): (a) Phase plane of $\left(e_{1}, \dot{e}_{1}\right)$. (b) Phase plane of $\left(e_{2}, \dot{e}_{2}\right)$. (c) The filtered tracking error $S_{1}$. (d) The filtered tracking error $S_{2}$.

where

$$
\begin{aligned}
& M_{11}=a_{1}+2 a_{3} \cos \left(q_{2}\right)+2 a_{4} \sin \left(q_{2}\right), \quad M_{22}=a_{2}, \\
& M_{21}=M_{12}=a_{2}+a_{3} \cos \left(q_{2}\right)+a_{4} \sin \left(q_{2}\right), \quad h=a_{3} \sin \left(q_{2}\right)-a_{4} \cos \left(q_{2}\right),
\end{aligned}
$$


a

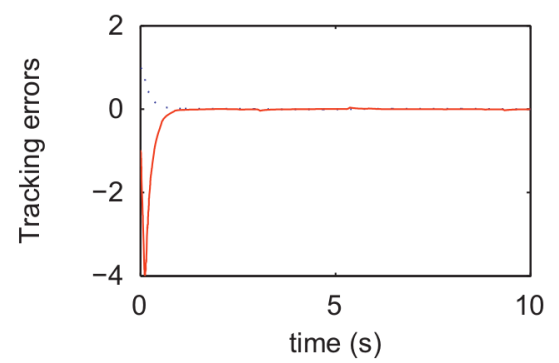

C

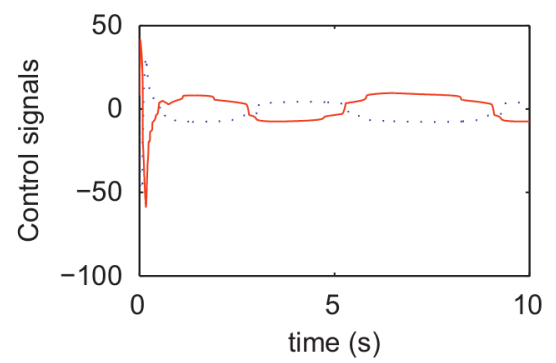

b

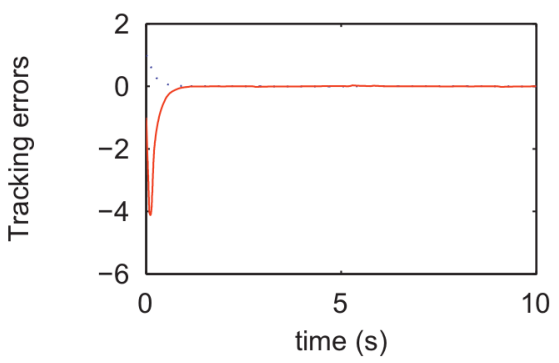

d

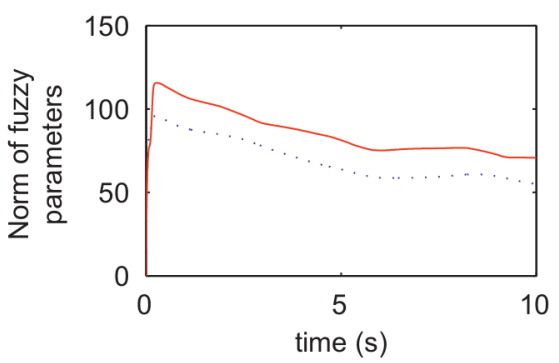

Fig. 4. Simulation results for example 1 (case 2): (a) Tracking errors: $e_{1}$ (dotted line) and $\dot{e}_{1}$ (solid line). (b) Tracking errors: $e_{2}$ (dotted line) and $\dot{e}_{2}$ (solid line). (c) Control input signals: $u_{1}$ (dotted line) and $u_{2}$ (solid line) (d) Norm of fuzzy parameters: $\left\|\theta_{1}\right\|$ (dotted line), $\left\|\theta_{2}\right\|($ solid line).

a

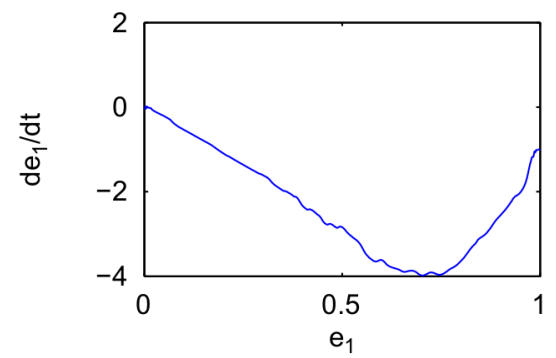

C

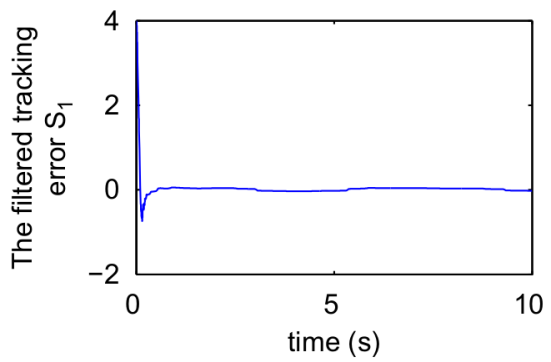

b

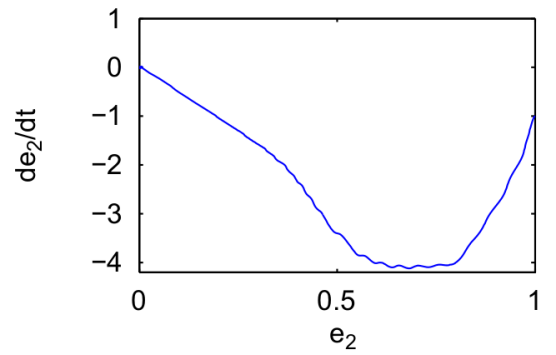

d

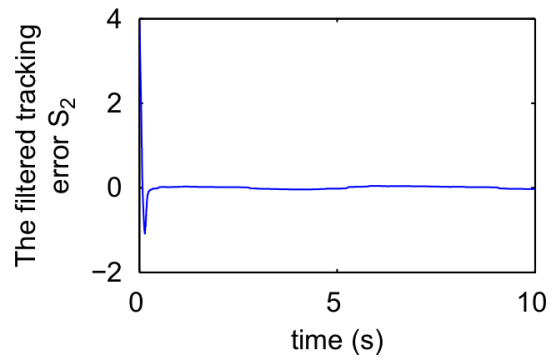

Fig. 5. Simulation results for example 1 (case 2): (a) Phase plane of $\left(e_{1}, \dot{e}_{1}\right)$. (b) Phase plane of $\left(e_{2}, \dot{e}_{2}\right)$. (c) The filtered tracking error $S_{1}$. (d) The filtered tracking error $S_{2}$.

with

$$
\begin{aligned}
& a_{1}=I_{1}+m_{1} l_{c 1}^{2}+I_{e}+m_{e} l_{c e}^{2}+m_{e} l_{1}^{2}, \quad a_{2}=I_{e}+m_{e} l_{c e}^{2}, \\
& a_{3}=m_{e} l_{1} l_{c e} \cos \left(\delta_{e}\right), \quad a_{4}=m_{e} l_{1} l_{c e} \sin \left(\delta_{e}\right) .
\end{aligned}
$$


The robot parameters are selected as follows:

$$
m_{1}=1, \quad m_{e}=2, \quad l_{1}=1, \quad l_{c 1}=0.5, \quad l_{c e}=0.6, \quad I_{1}=0.12, \quad I_{e}=0.25, \quad \delta_{e}=30^{\circ}
$$

a

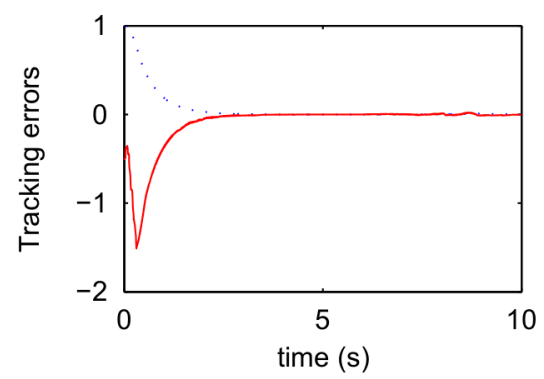

C

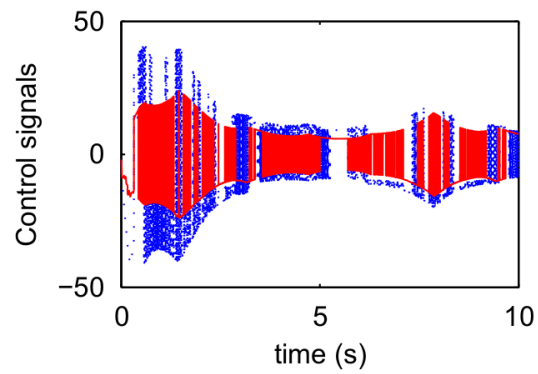

b

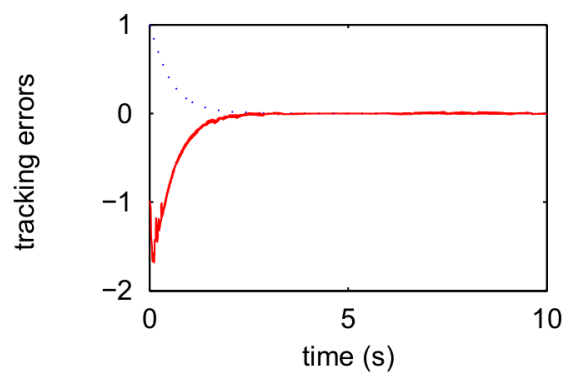

d

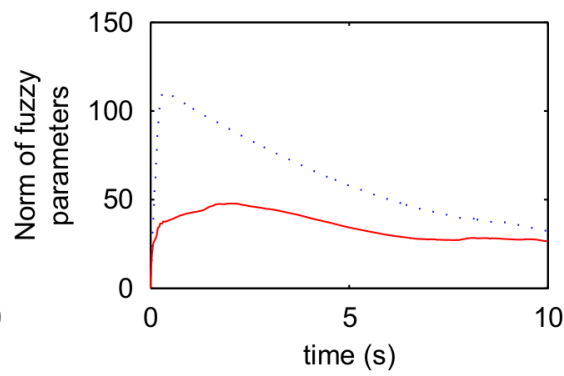

Fig. 6. Simulation results for example 2 (case 1): (a) Tracking errors: $e_{1}$ (dotted line) and $\dot{e}_{1}$ (solid line). (b) Tracking errors: $e_{2}$ (dotted line) and $\dot{e}_{2}$ (solid line). (c) Control input signals: $u_{1}$ (dotted line) and $u_{2}$ (solid line). (d) Norm of fuzzy parameters: $\left\|\theta_{1}\right\|$ (dotted line), $\left\|\theta_{2}\right\|$ (solid line).

a

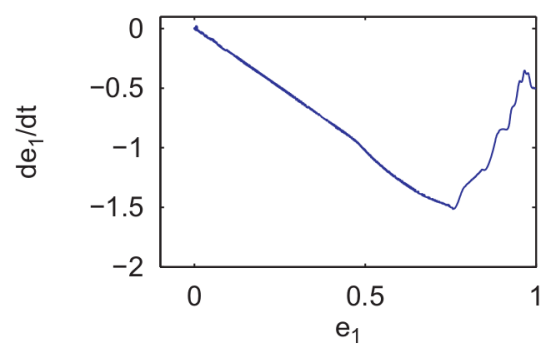

C

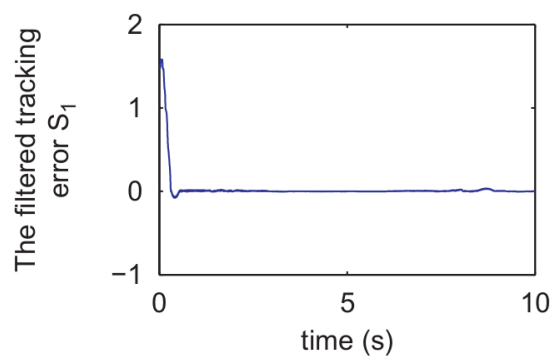

b

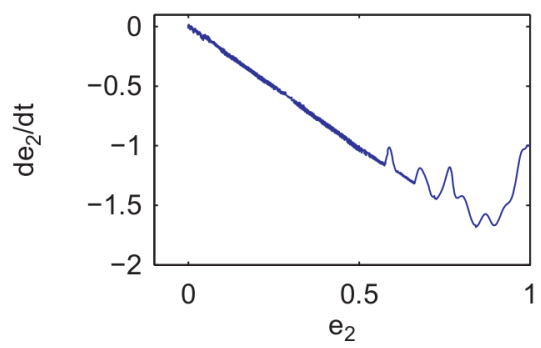

d

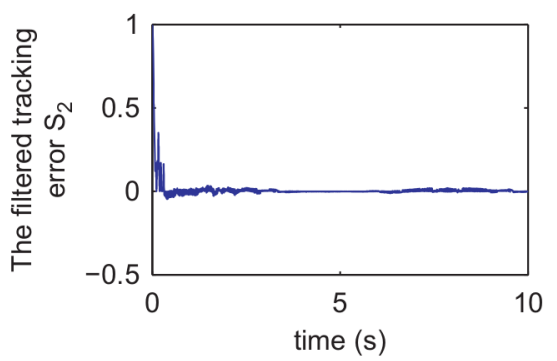

Fig. 7. Simulation results for example 2 (case 1): (a) Phase plane of $\left(e_{1}, \dot{e}_{1}\right)$. (b) Phase plane of $\left(e_{2}, \dot{e}_{2}\right)$. (c) The filtered tracking error $S_{1}$. (d) The filtered tracking error $S_{2}$. 
Let $\left[y_{1}, y_{2}\right]=\left[q_{1}, q_{2}\right], u=\left[u_{1}, u_{2}\right]^{T}, \Phi(u)=\left[\Phi_{1}\left(u_{1}\right), \Phi_{2}\left(u_{2}\right)\right]^{T}$, and $x=\left[x_{11}, x_{12}, x_{21}, x_{22}\right]^{T}=\left[q_{1}, \dot{q}_{1}, q_{2}, \dot{q}_{2}\right]^{T}$. We can rewrite the robot model with actuator nonlinearities as follows:

$$
\ddot{y}=F(x)+G(x) \Phi(u),
$$

a

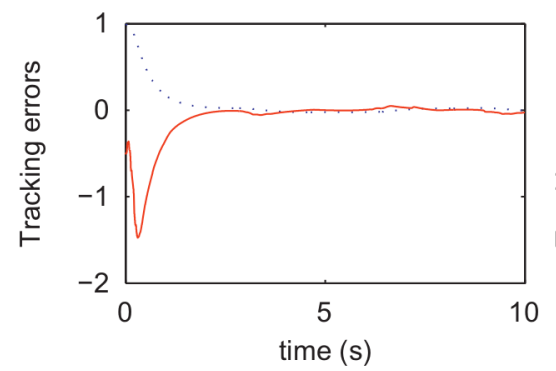

C

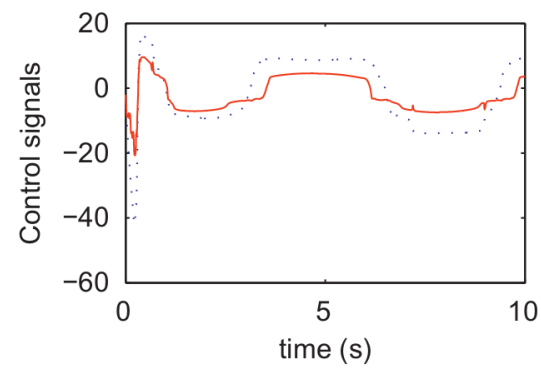

b

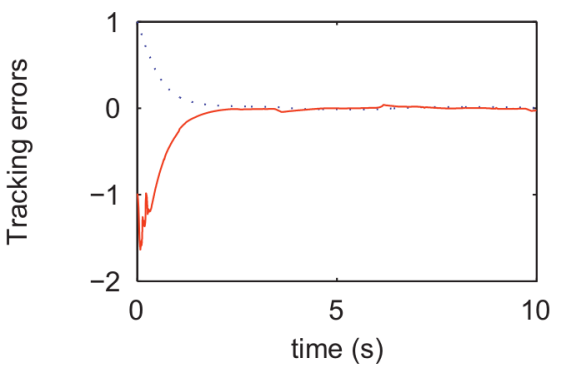

d

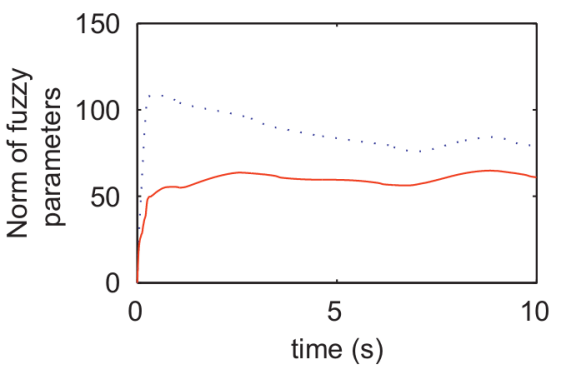

Fig. 8. Simulation results for example 2 (case 2): (a) Tracking errors: $e_{1}$ (dotted line) and $\dot{e}_{1}$ (solid line). (b) Tracking errors: $e_{2}$ (dotted line) and $\dot{e}_{2}$ (solid line). (c) Control input signals: $u_{1}$ (dotted line) and $u_{2}$ (solid line). (d) Norm of fuzzy parameters: $\left\|\theta_{1}\right\|$ (dotted line), $\left\|\theta_{2}\right\|$ (solid line).

a

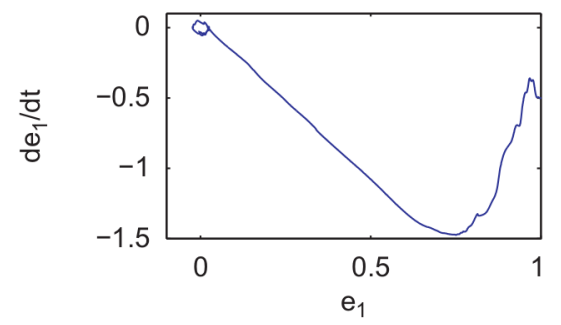

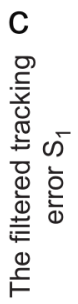

b

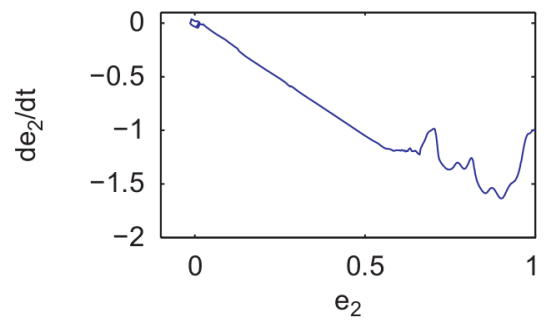

d

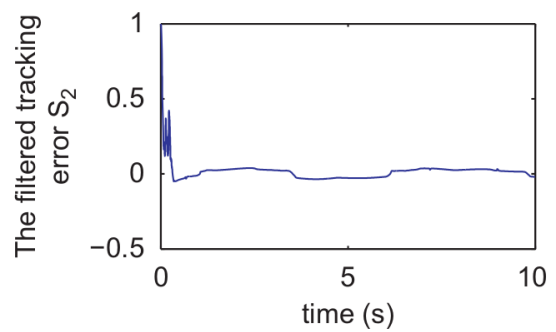

Fig. 9. Simulation results for example 2 (case 2): (a) Phase plane of $\left(e_{1}, \dot{e}_{1}\right)$. (b) Phase plane of ( $\left.e_{2}, \dot{e}_{2}\right)$. (c) The filtered tracking error $S_{1}$. (d) The filtered tracking error $S_{2}$. 
where

$$
\begin{aligned}
& F(x)=\left(\begin{array}{l}
f_{1}(x) \\
f_{2}(x)
\end{array}\right)=-M^{-1}\left(\begin{array}{cc}
-h \dot{q}_{2} & -h\left(\dot{q}_{1}+\dot{q}_{2}\right) \\
h \dot{q}_{1} & 0
\end{array}\right)\left(\begin{array}{l}
\dot{q}_{1} \\
\dot{q}_{2}
\end{array}\right), \\
& G(x)=\left(\begin{array}{ll}
g_{11}(x) & g_{12}(x) \\
g_{21}(x) & g_{22}(x)
\end{array}\right)=M^{-1}=\left(\begin{array}{ll}
M_{11} & M_{12} \\
M_{21} & M_{22}
\end{array}\right)^{-1} .
\end{aligned}
$$

The input nonlinearities $\Phi_{i}\left(u_{i}\right)$ for $i=1,2$ are described by (51).

The control objective consists in allowing the system outputs $q_{1}$ and $q_{2}$ to, respectively, track the sinusoidal desired trajectories $y_{d 1}=\sin (t)$ and $y_{d 2}=\sin (t)$. The fuzzy system $\theta_{1}^{T} \psi_{1}\left(\bar{z}_{1}\right)$ has the vector $\left[x^{T}, u_{2}\right]^{T}$ as input, while the fuzzy system $\theta_{2}^{T} \psi_{2}\left(\bar{z}_{2}\right)$ has the state vector $x$ as input. For each variable of the entries of these fuzzy systems, as in [2], we define three (one triangular and two trapezoidal) membership functions uniformly distributed on the intervals $[-2,2]$ for $x_{11}, x_{12}, x_{21}$, and $x_{22}$, and $[-25,25]$ for $u_{2}$. The design parameters used in this simulation are chosen as follows: $\gamma_{01}=\gamma_{02}=20, \gamma_{11}=20, \gamma_{12}=20, \gamma_{21}=1500, \gamma_{22}=1500, \sigma_{01}=\sigma_{02}=0.001, \sigma_{11}=\sigma_{12}=0.001$, $\sigma_{21}=0.0001, \sigma_{22}=0.0001, \lambda_{1}=\lambda_{2}=2, k_{21}=k_{22}=2$. The initial conditions are selected as: $x(0)=\left[\begin{array}{llll}1 & 0.5 & 1 & 0\end{array}\right]$, $k_{01}(0)=k_{02}(0)=0.01, k_{11}(0)=k_{12}(0)=0.01, \theta_{1 i}(0)=\theta_{2 i}(0)=0.001$.

As in the previous example, the following cases are considered to illustrate the effectiveness of the proposed controller:

- The discontinuous controller (48) is applied to the system (53).

- A practical (smooth) version of the controller (48) is applied to the system (53).

(a) Simulation results with the discontinuous function $\operatorname{sign}\left(\bar{S}_{i}\right)$ : The simulation results are depicted in Figs. 6 and 7. In spite of the presence of the input nonlinearities, these results show a good tracking performance. From Fig. 7, for both links, it is seen that a relatively quick reaching phase over the sliding line is achieved. When the line is reached, the sliding behaviour is observed and the tracking error decays to zero with the error dynamics dictated by the parameter $\lambda_{i}$ of the controller (sliding lines slope is $-\lambda_{i}=-2$ ).

(b) Simulation results with the smooth function $\tanh \left(k_{s i} \bar{S}_{i}\right)$ : Using a smooth function $\left(\tanh \left(k_{s i} \bar{S}_{i}\right)\right.$, with $\left.k_{s i}=10\right)$, the obtained results are shown in Figs. 8 and 9.

\section{Conclusion}

In this paper, a fuzzy adaptive variable-structure controller for a class of MIMO unknown nonlinear time-delays systems subject to actuator sector nonlinearities and dead-zones has been proposed bearing in mind the stability and convergence insights. A suitable decomposition of the control-gain matrix has been fully exploited to carry out the controller design and the stability analysis. The uncertainties from unknown time-varying delays have been compensated for through the use of an appropriate Lyapunov-Krasovskii functional. Of fundamental interest, it has been shown that the underlying control system is stable and that the involved tracking errors exponentially converge to an adjustable region. Probing simulation results have been given to emphasize the effectiveness of the proposed controller.

\section{Appendix A}

Proof of Theorem 1. Let us consider the following Lyapunov-Krasovskii functional:

$$
V=V_{1}+V_{2} \text {, }
$$

where

$$
\begin{aligned}
& V_{1}=\frac{1}{2 \eta} \bar{S}^{T} G_{s 2}(x) \bar{S}+\frac{1}{2} \sum_{i=1}^{p} \frac{1}{\gamma_{0 i}}\left(\tilde{k}_{0 i}\right)^{2}+\frac{1}{2} \sum_{i=1}^{p} \frac{1}{\gamma_{1 i}}\left(\tilde{k}_{1 i}\right)^{2}+\frac{1}{2} \sum_{i=1}^{p} \frac{1}{\gamma_{2 i}} \tilde{\theta}_{i}^{T} \tilde{\theta}_{i}, \\
& V_{2}=\frac{1}{\eta} \sum_{i=1}^{p} \bar{E}_{i}^{T} P_{i} \bar{E}_{i}+\frac{l p}{2 \eta} \sum_{i=1}^{p}\left(\frac{e^{\omega \tau_{i}^{*}}}{\left(1-\bar{\tau}_{i}\right)} \int_{t-\tau_{i}(t)}^{t} e^{-\omega(t-s)}\left\|\bar{E}_{i}(s)\right\|^{2} d s\right) .
\end{aligned}
$$


The time derivative of $V_{1}$ is given by

$$
\dot{V}_{1}=\frac{1}{\eta} \bar{S}^{T} G_{s 2}(x) \dot{\bar{S}}+\frac{1}{2 \eta} \bar{S}^{T} \dot{G}_{s 2} \bar{S}+\sum_{i=1}^{p} \frac{1}{\gamma_{0 i}} \tilde{k}_{0 i} \dot{k}_{0 i}+\sum_{i=1}^{p} \frac{1}{\gamma_{1 i}} \tilde{k}_{1 i} \dot{k}_{1 i}+\sum_{i=1}^{p} \frac{1}{\gamma_{2 i}} \tilde{\theta}_{i}^{T} \dot{\theta}_{i} .
$$

Before continuing the stability analysis, we must calculate the upper bound of $\bar{S}_{i} \Phi_{i}\left(u_{i}\right)$. We can easily show from (42) that $u_{i}<-u_{i-}$ for $\bar{S}_{i}>0$ and $u_{i}>u_{i+}$ for $\bar{S}_{i}<0$. This makes it possible to conclude from (25) and (42) that for $\bar{S}_{i}>0$ we have

$$
\left(u_{i}+u_{i-}\right) \Phi_{i}\left(u_{i}\right)=-\rho_{i}(t) \operatorname{sign}\left(\bar{S}_{i}\right) \Phi_{i}\left(u_{i}\right) \geq m_{i-}^{*} \rho_{i}^{2}(t)\left[\operatorname{sign}\left(\bar{S}_{i}\right)\right]^{2} \geq \eta \rho_{i}^{2}(t),
$$

and for $\bar{S}_{i}<0$ we have

$$
\left(u_{i}-u_{i+}\right) \Phi_{i}\left(u_{i}\right)=-\rho_{i}(t) \operatorname{sign}\left(\bar{S}_{i}\right) \Phi_{i}\left(u_{i}\right) \geq m_{i+}^{*} \rho_{i}^{2}(t)\left[\operatorname{sign}\left(\bar{S}_{i}\right)\right]^{2} \geq \eta \rho_{i}^{2}(t) .
$$

Then, for $\bar{S}_{i}<0$ and $\bar{S}_{i}>0$ we have

$$
-\rho_{i}(t) \operatorname{sign}\left(\bar{S}_{i}\right) \Phi_{i}\left(u_{i}\right) \geq \eta \rho_{i}^{2}(t) .
$$

And using (A.7) and the fact that $\bar{S}_{i}^{2}>0$ and $\bar{S}_{i} \operatorname{sign}\left(\bar{S}_{i}\right)=\left|\bar{S}_{i}\right|$ yields

$$
-\rho_{i}(t) \bar{S}_{i}^{2} \operatorname{sign}\left(\bar{S}_{i}\right) \Phi_{i}\left(u_{i}\right) \geq \eta \rho_{i}^{2}(t) \bar{S}_{i}^{2}=\eta \rho_{i}^{2}(t)\left|\bar{S}_{i}\right|^{2} .
$$

Finally for all $\bar{S}_{i}$ (i.e. for $\bar{S}_{i}<0, \bar{S}_{i}=0$ and $\bar{S}_{i}>0$ ) we have

$$
\bar{S}_{i} \Phi_{i}\left(u_{i}\right) \leq-\eta \rho_{i}(t)\left|\bar{S}_{i}\right| .
$$

And using the expressions (43)-(45), (A.9) and (47), (A.4) becomes

$$
\begin{aligned}
\dot{V}_{1} \leq & \sum_{i=1}^{p}\left|\bar{S}_{i}\right|\left(k_{0 i}+k_{1 i}\left|\bar{S}_{i}\right|+\theta_{i}^{T} \psi_{i}\left(\bar{z}_{i}\right)\right)+\frac{1}{\eta} \bar{S}^{T} \Phi(u)-\sum_{i=1}^{p} \frac{\left\|P_{i} B_{i}\right\|^{2}}{\eta} \bar{S}_{i}^{2}+\frac{l p}{2 \eta}\left\|\bar{E}_{\tau}\right\|^{2}-\sum_{i=1}^{p} \sigma_{0 i} \tilde{k}_{0 i} k_{0 i} \\
& -\sum_{i=1}^{p} \sigma_{1 i} \tilde{k}_{1 i} k_{1 i}-\sum_{i=1}^{p} \sigma_{2 i} \tilde{\theta}_{i}^{T} \theta_{i} \\
\leq & -\sum_{i=1}^{p} k_{2 i} \bar{S}_{i}^{2}-\sum_{i=1}^{p} \frac{\left\|P_{i} B_{i}\right\|^{2}}{\eta} \bar{S}_{i}^{2}+\frac{l p}{2 \eta}\left\|\bar{E}_{\tau}\right\|^{2}-\sum_{i=1}^{p} \sigma_{0 i} \tilde{k}_{0 i} k_{0 i}-\sum_{i=1}^{p} \sigma_{1 i} \tilde{k}_{1 i} k_{1 i}-\sum_{i=1}^{p} \sigma_{2 i} \tilde{\theta}_{i}^{T} \theta_{i} .
\end{aligned}
$$

We can easily check that

$$
\begin{aligned}
& -\sigma_{0 i} \tilde{k}_{0 i} k_{0 i} \leq-\frac{\sigma_{0 i}}{2} \tilde{k}_{0 i}^{2}+\frac{\sigma_{0 i}}{2} k_{0 i}^{* 2}, \\
& -\sigma_{1 i} \tilde{k}_{1 i} k_{1 i} \leq-\frac{\sigma_{1 i}}{2} \tilde{k}_{1 i}^{2}+\frac{\sigma_{1 i}}{2} k_{1 i}^{* 2}, \\
& -\sigma_{2 i} \tilde{\theta}_{i}^{T} \theta_{i} \leq-\frac{\sigma_{2 i}}{2}\left\|\tilde{\theta}_{i}\right\|^{2}+\frac{\sigma_{2 i}}{2}\left\|\theta_{i}^{*}\right\|^{2} .
\end{aligned}
$$

And using the previous inequalities, (A.10) becomes

$$
\dot{V}_{1} \leq-\sum_{i=1}^{p} k_{2 i} \bar{S}_{i}^{2}-\sum_{i=1}^{p} \frac{\sigma_{0 i}}{2} \tilde{k}_{0 i}^{2}-\sum_{i=1}^{p} \frac{\sigma_{1 i}}{2} \tilde{k}_{1 i}^{2}-\sum_{i=1}^{p} \frac{\sigma_{2 i}}{2}\left\|\tilde{\theta}_{i}\right\|^{2}-\sum_{i=1}^{p} \frac{\left\|P_{i} B_{i}\right\|^{2}}{\eta} \bar{S}_{i}^{2}+\frac{l p}{2 \eta}\left\|\bar{E}_{\tau}\right\|^{2}+\pi,
$$

where

$$
\pi=\sum_{i=1}^{p} \frac{\sigma_{0 i}}{2} k_{0 i}^{* 2}+\sum_{i=1}^{p} \frac{\sigma_{1 i}}{2} k_{1 i}^{* 2}+\sum_{i=1}^{p} \frac{\sigma_{2 i}}{2}\left\|\theta_{i}^{*}\right\|^{2} .
$$


The time derivative of $V_{2}$ is given by

$$
\begin{aligned}
\dot{V}_{2}= & -\frac{1}{\eta} \sum_{i=1}^{p} \bar{E}_{i}^{T} Q_{i} \bar{E}_{i}+\frac{2}{\eta} \sum_{i=1}^{p} \bar{E}_{i}^{T} P_{i} B_{i} S_{i}-\omega \frac{l p}{2 \eta} \sum_{i=1}^{p}\left(\frac{e^{\omega \tau_{i}^{*}}}{\left(1-\bar{\tau}_{i}\right)} \int_{t-\tau_{i}(t)}^{t} e^{-\omega(t-s)}\left\|\bar{E}_{i}(s)\right\|^{2} d s\right) \\
& +\frac{l p}{2 \eta} \sum_{i=1}^{p} \frac{e^{\omega \tau_{i}^{*}}}{\left(1-\bar{\tau}_{i}\right)}\left\|\bar{E}_{i}(t)\right\|^{2}-\frac{l p}{2 \eta} \sum_{i=1}^{p} \frac{1-\dot{\tau}_{i}(t)}{\left(1-\bar{\tau}_{i}\right)} e^{\omega\left(\tau_{i}^{*}-\tau_{i}(t)\right)}\left\|\bar{E}_{i}\left(t-\tau_{i}(t)\right)\right\|^{2}
\end{aligned}
$$

Using the following properties:

$$
\begin{aligned}
& 2 \bar{E}_{i}^{T} P_{i} B_{i} S_{i} \leq \frac{\left\|\bar{E}_{i}\right\|^{2}\left\|P_{i} B_{i}\right\|^{2}}{\left\|P_{i} B_{i}\right\|^{2}}+\left\|P_{i} B_{i}\right\|^{2}\left\|\bar{S}_{i}\right\|^{2}=\left\|\bar{E}_{i}\right\|^{2}+\left\|P_{i} B_{i}\right\|^{2}\left\|\bar{S}_{i}\right\|^{2}, \\
& \frac{1-\dot{\tau}_{i}(t)}{\left(1-\bar{\tau}_{i}\right)} e^{\omega\left(\tau_{i}^{*}-\tau_{i}(t)\right)} \geq 1, \quad \sum_{i=1}^{p} \frac{l e^{\omega \tau_{i}^{*}}}{\left(1-\bar{\tau}_{i}\right)}\left\|\bar{E}_{i}(t)\right\|^{2} \leq \sum_{i=1}^{p}\left\|\bar{E}_{i}(t)\right\|^{2}=\sum_{i=1}^{p}\left\|\bar{E}_{i}\right\|^{2}, \text { and } \\
& \lambda_{\min }\left(Q_{i}\right)\left\|\bar{E}_{i}\right\|^{2} \leq \bar{E}_{i}^{T} Q_{i} \bar{E}_{i} \leq \lambda_{\max }\left(Q_{i}\right)\left\|\bar{E}_{i}\right\|^{2} .
\end{aligned}
$$

(A.12) becomes

$$
\begin{aligned}
\dot{V}_{2} \leq & -\frac{1}{\eta} \sum_{i=1}^{p}\left(\lambda_{\min }\left(Q_{i}\right)-1\right)\left\|\bar{E}_{i}\right\|^{2}+\sum_{i=1}^{p} \frac{\left\|P_{i} B_{i}\right\|^{2}}{\eta} \bar{S}_{i}^{2}-\omega \frac{l p}{2 \eta} \sum_{i=1}^{p}\left(\frac{e^{\omega \tau_{i}^{*}}}{\left(1-\bar{\tau}_{i}\right)} \int_{t-\tau_{i}(t)}^{t} e^{-\omega(t-s)}\left\|\bar{E}_{i}(s)\right\|^{2} d s\right) \\
& +\frac{p}{2 \eta} \sum_{i=1}^{p}\left\|\bar{E}_{i}\right\|^{2}-\frac{l p}{2 \eta} \sum_{i=1}^{p} \frac{1-\dot{\tau}_{i}(t)}{\left(1-\bar{\tau}_{i}\right)} e^{\omega\left(\tau_{i}^{*}-\tau_{i}(t)\right)}\left\|\bar{E}_{i}\left(t-\tau_{i}(t)\right)\right\|^{2} \\
= & -\frac{1}{\eta} \sum_{i=1}^{p}\left(\lambda_{\min }\left(Q_{i}\right)-(0.5 p+1)\right)\left\|\bar{E}_{i}\right\|^{2}-\omega \frac{l p}{2 \eta} \sum_{i=1}^{p}\left(\frac{e^{\omega \tau_{i}^{*}}}{\left(1-\bar{\tau}_{i}\right)} \int_{t-\tau_{i}(t)}^{t} e^{-\omega(t-s)}\left\|\bar{E}_{i}(s)\right\|^{2} d s\right) \\
& +\sum_{i=1}^{p} \frac{\left\|P_{i} B_{i}\right\|^{2}}{\eta} \bar{S}_{i}^{2}-\frac{l p}{2 \eta}\left\|\bar{E}_{\tau}\right\|^{2} .
\end{aligned}
$$

Accordingly, we obtain

$$
\begin{aligned}
\dot{V}= & \dot{V}_{1}+\dot{V}_{2} \leq-\sum_{i=1}^{p} k_{2 i} \bar{S}_{i}^{2}-\sum_{i=1}^{p} \frac{\sigma_{0 i}}{2} \tilde{k}_{0 i}^{2}-\sum_{i=1}^{p} \frac{\sigma_{1 i}}{2} \tilde{k}_{1 i}^{2}-\sum_{i=1}^{p} \frac{\sigma_{2 i}}{2}\left\|\tilde{\theta}_{i}\right\|^{2}-\frac{1}{\eta} \sum_{i=1}^{p}\left(\lambda_{\min }\left(Q_{i}\right)-(0.5 p+1)\right)\left\|\bar{E}_{i}\right\|^{2} \\
& -\omega \frac{l p}{2 \eta} \sum_{i=1}^{p}\left(\frac{e^{\omega \tau_{i}^{*}}}{\left(1-\bar{\tau}_{i}\right)} \int_{t-\tau_{i}(t)}^{t} e^{-\omega(t-s)}\left\|\bar{E}_{i}(s)\right\|^{2} d s\right)+\pi .
\end{aligned}
$$

Since $G_{s}(x)$ is symmetric and positive-definite, thus there exists a positive scalar $\sigma_{g 0}$ such that $G_{s}(x) \geq \sigma_{g 0} I_{p}$ and henceforth

$$
\bar{S}^{T} G_{s 2}(x) \bar{S}=S^{T} G_{s}^{-1}(x) S \leq \frac{1}{\sigma_{g 0}}\|S\|^{2}=\frac{1}{\sigma_{g 0}}\|\bar{S}\|^{2} .
$$

Recall that $D=D^{T}=D^{-1}$ and $\bar{S}=D^{-1} S$.

And using (A.14) and (A.15) yields

$$
\dot{V} \leq-\mu V+\pi,
$$

where

$$
\mu=\omega=\min \left\{\min _{i}\left\{2 \eta \sigma_{g 0} k_{1 i}\right\}, \min _{i}\left\{\frac{\lambda_{\min }\left(Q_{i}\right)-(0.5 p+1)}{\lambda_{\max }\left(P_{i}\right)}\right\}, \min _{i}\left\{\gamma_{0 i} \sigma_{0 i}\right\}, \min _{i}\left\{\gamma_{1 i} \sigma_{1 i}\right\}, \min _{i}\left\{\gamma_{2 i} \sigma_{2 i}\right\}\right\} .
$$


Multiplying (A.16) by $e^{\mu t}$ yields

$$
\frac{d}{d t}\left(V e^{\mu t}\right) \leq \pi e^{\mu t}
$$

Integrating (A.17) over $[0, t]$, we have

$$
0 \leq V(t) \leq \frac{\pi}{\mu}+\left(V(0)-\frac{\pi}{\mu}\right) e^{-\mu t}
$$

Therefore, all variables of the control system, i.e. $k_{0 i}, k_{1 i}, \theta_{i}, S_{i}, \bar{E}_{i}$ and $x$, are uniformly ultimately bounded. And hence the input $u_{i}$ is bounded.

With bearing in mind of (A.1)-(A.3), we can define $V(0)$ as follows:

$$
\begin{aligned}
V(0)= & \frac{1}{2 \eta} \bar{S}(0)^{T} G_{s 2}(x(0)) \bar{S}(0)+\frac{1}{2} \sum_{i=1}^{p} \frac{1}{\gamma_{0 i}}\left(k_{0 i}(0)-k_{0 i}^{*}\right)^{2}+\frac{1}{2} \sum_{i=1}^{p} \frac{1}{\gamma_{1 i}}\left(k_{1 i}(0)-k_{1 i}^{*}\right)^{2} \\
& +\frac{1}{2} \sum_{i=1}^{p} \frac{1}{\gamma_{2 i}}\left(\theta_{i}(0)-\theta_{i}^{*}\right)^{T}\left(\theta_{i}(0)-\theta_{i}^{*}\right)+\frac{1}{\eta} \sum_{i=1}^{p} \bar{E}_{i}^{T}(0) P_{i} \bar{E}_{i}(0) \\
& +\frac{l p}{2 \eta} \sum_{i=1}^{p}\left(\frac{e^{\omega \tau_{i}^{*}}}{\left(1-\bar{\tau}_{i}\right)} \int_{0-\tau_{i}(0)}^{0} e^{\omega s}\left\|\bar{E}_{i}(s)\right\|^{2} d s\right) .
\end{aligned}
$$

Since $G_{s 2}(x)$ is symmetric and positive-definite, i.e. there exists an unknown positive constant $\sigma_{g 2}$ such that $G_{s 2}(x) \geq$ $\sigma_{g 2} I_{p}$, it follows from (A.18) and (A.1) that

$$
\left|S_{i}\right| \leq\left(\frac{2 \eta}{\sigma_{g 2}}\left(\frac{\pi}{\mu}+\left(V(0)-\frac{\pi}{\mu}\right) e^{-\mu t}\right)\right)^{1 / 2} .
$$

Then, the solution of $S_{i}$ exponentially converges to a bounded adjustable domain defined as follows:

$$
\Omega_{S_{i}}=\left\{S_{i}|| S_{i} \mid \leq\left(\frac{2 \eta \pi}{\sigma_{g 2} \mu}\right)^{1 / 2}\right\} .
$$

From (A.18) and (A.1), we can also easily show that the tracking error exponentially converges to the following bounded region:

$$
\Omega_{\bar{E}_{i}}=\left\{\bar{E}_{i} \mid\left\|\bar{E}_{i}\right\| \leq\left(\frac{\eta}{\lambda_{\min }\left(P_{i}\right)} \frac{\pi}{\mu}\right)^{1 / 2}\right\} .
$$

This ends the proof of the theorem.

\section{References}

[1] A. Boulkroune, M. M'Saad, M. Tadjine, M. Farza, Adaptive fuzzy control for MIMO nonlinear systems with unknown dead-zone, in: Proc. of the Fourth Internat. IEEE Conf. on Intelligent Systems, Varna, Bulgaria, September 2008, pp. 450-455.

[2] A. Boulkroune, M. Tadjine, M. M'saad, M. Farza, How to design a fuzzy adaptive control based on observers for uncertain affine nonlinear systems, Fuzzy Sets and Systems 159 (2008) 926-948.

[3] A. Boulkroune, M. M'Saad, M. Tadjine, M. Farza, Fuzzy adaptive controller for MIMO nonlinear systems with known and unknown control direction, Fuzzy Sets and Systems 161 (2010) 797-820.

[4] A. Boulkroune, M. Tadjine, M. M'saad, M. Farza, Adaptive fuzzy controller for non-affine systems with zero dynamics, International Journal of Systems Science 40 (4) (2009) 367-382.

[5] Y.C. Chang, Robust tracking control for nonlinear MIMO systems via fuzzy approaches, Automatica 36 (2000) 1535-1545.

[6] H. Chekireb, M. Tadjine, D. Bouchaffra, Direct adaptive fuzzy control of nonlinear system class with applications, Control and Intelligent Systems 31 (2) (2003) 1-11.

[7] J. Chen, A. Behal, D.M. Dawson, Adaptive output feedback control for a class of MIMO nonlinear systems, in: Proc. of the American Control Conf., Minneapolis, MN, June 2006, pp. 5300-5305. 
[8] R.R. Costa, Li. Hsu, A.K. Imai, P. Kokotovic, Lyapunov-based adaptive control of MIMO systems, Automatica 39 (7) (2003) $1251-1257$.

[9] N. Essounbouli, A. Hamzaoui, J. Zaytoon, An improved robust adaptive fuzzy controller for MIMO systems, Control and Intelligent Systems 34 (1) (2006) 12-21.

[10] S.S. Ge, F. Hong, T.H. Lee, Adaptive neural network control of nonlinear systems with unknown time delays, IEEE Transactions on Automatic Control 48 (11) (2003) 2004-2010.

[11] S.S. Ge, F. Hong, T.H. Lee, Adaptive neural control of nonlinear time-delay system with unknown virtual control coefficients, IEEE Transactions on Systems, Man, and Cybernetics_-Part B: Cybernetics 34 (1) (2004) 499-516.

[12] S.S. Ge, K.P. Tee, Approximation-based control of nonlinear MIMO time-delay systems, Automatica 43 (2007) 31-43.

[13] N. Golea, A. Golea, K. Benmahammed, Stable indirect fuzzy adaptive control, Fuzzy Sets and Systems 137 (2003) $353-366$.

[14] K. Gu, V.L. Kharitonov, J. Chen, Stability of Time-delay Systems, Birkhauser, Boston, 2003.

[15] H.M. Gutierrez, P.I. Ro, Sliding-mode control of a nonlinear-input system: application to a magnetically levitated fast-tool servo, IEEE Transactions on Industrial Electronics 45 (1998) 921-927.

[16] K.-C. Hsu, Decentralized sliding mode controller for uncertain time-delayed systems with series nonlinearities, ASME Journal of Dynamic Systems, Measurement and Control 121 (4) (1999) 708-713.

[17] K.-C. Hsu, W.-Y. Wang, P.-Z. Lin, Sliding mode control for uncertain nonlinear systems with multiple inputs containing sector nonlinearities and deadzones, IEEE Transactions on Systems, Man and Cybernetic, Part-B 34 (1) (2004) 374-380.

[18] L. Hsu, R.R. Costa, F. Lizarralde, Lyapunov/passivity-based adaptive control of relative degree two MIMO systems with an application to visual servoing, IEEE Transactions on Automatic Control 52 (2) (2007) 364-371.

[19] V.L. Kharitonov, D. Melchor-Aguilar, Lyapunov-Krasovskii functionals for additional dynamics, International Journal of Robust Nonlinear Control 13 (2003) 793-804.

[20] V.B. Kolmanovskii, J. Richard, Stability of some linear systems with delays, IEEE Transactions on Automatic Control 44 (5) (1999) 984-989.

[21] S. Labiod, M.S. Boucherit, T.M. Guerra, Adaptive fuzzy control of a class of MIMO nonlinear systems, Fuzzy Sets and Systems 151 (2005) $59-77$.

[22] H.-X. Li, S.C. Tong, A hybrid adaptive fuzzy control for a class of nonlinear MIMO systems, IEEE Transactions on Fuzzy Systems 11 (1) (2003) 24-34.

[23] Y. Niu, D.W.C. Ho, Design of sliding mode control for nonlinear stochastic systems subject to actuator nonlinearity, IEE Proceedings-Control Theory and Applications 153 (6) (2006) 737-744.

[24] R. Ordonez, K.M. Passino, Stable multi-input multi-output adaptive fuzzy/neural control, IEEE Transactions on Fuzzy Systems 7 (3) (1999) $345-353$.

[25] K.-K. Shyu, W.-J. Liu, K.-C. Hsu, Decentralized variable structure control design for uncertain large scale systems containing a deadzone, IEE Proceedings-Control Theory and Applications 150 (5) (2003) 467-475.

[26] K.-K. Shyu, W.-J. Liu, K.-C. Hsu, Design of large-scale time-delayed systems with dead-zone input via variable structure control, Automatica 41 (2005) 1239-1246.

[27] G. Strang, Linear Algebra and its Applications, second ed., Academic Press, New York, 1980.

[28] Y. Sun, J. Hsieh, H. Yang, On the stability of uncertain systems with multiple time-varying delays, IEEE Transactions on Automatic Control 42 (1) (1997) 101-105.

[29] S.C. Tong, J. Tang, T. Wang, Fuzzy adaptive control of multivariable nonlinear systems, Fuzzy Sets and Systems 111 (2) (2000) $153-167$.

[30] S.C. Tong, H.X. Li, Fuzzy adaptive sliding model control for MIMO nonlinear systems, IEEE Transactions on Fuzzy Systems 11 (3) (2003) $354-360$.

[31] S.C. Tong, B. Chen, Y. Wang, Fuzzy adaptive output feedback control for MIMO nonlinear systems, Fuzzy Sets and Systems 156 (2) (2005) 285-299.

[32] M. Wang, B. Chen, K. Liu, X. Liu, S. Zhang, Adaptive fuzzy tracking control of nonlinear time-delay systems with unknown virtual control coefficients, Information Sciences 178 (2008) 4326-4340.

[33] L.X. Wang, Adaptive Fuzzy Systems and Control: Design and Stability Analysis, Prentice-Hall, Englewood Cliffs, NJ, 1994.

[34] T.P. Zhang, S.S. Ge, Adaptive neural control of MIMO nonlinear state time-varying delay systems with unknown dead-zones and gain signs, Automatica 43 (6) (2007) 1021-1033.

[35] T.P. Zhang, Y. Yi, Adaptive fuzzy control for a class of MIMO nonlinear systems with unknown dead-zones, Acta Automatica Sinica 33 (1) (2007) 96-99.

[36] X.T. Zhang, D.M. Dawson, M.S. De Queiroz, B. Xian, Adaptive control for a class of MIMO nonlinear systems with non-symmetric input matrix, in: Proc. of the IEEE Internat. Conf. on Control Applications, Taipei, Taiwan, September 2004, pp. 1324-1329. 